\title{
Toward Translating Raw Indoor Positioning Data into Mobility Semantics
}

\author{
HUAN LI and HUA LU, Aalborg University, Denmark \\ GANG CHEN, KE CHEN, QINKUANG CHEN, and LIDAN SHOU*, Zhejiang University, China
}

\begin{abstract}
Indoor mobility analyses are increasingly interesting due to the rapid growth of raw indoor positioning data obtained from IoT infrastructure. However, high-level analyses are still in urgent need of a concise but semantics-oriented representation of the mobility implied by the raw data. This work studies the problem of translating raw indoor positioning data into mobility semantics that describe a moving object's mobility event (What) someplace (Where) at some time (When). The problem is non-trivial mainly because of the inherent errors in the uncertain, discrete raw data. We propose a three-layer framework to tackle the problem. In the cleaning layer, we design a cleaning method that eliminates positioning data errors by considering indoor mobility constraints. In the annotation layer, we propose a split-and-match approach to annotate mobility semantics on the cleaned data. The approach first employs a density-based splitting method to divide positioning sequences into split snippets according to underlying mobility events, followed by a semantic matching method that makes proper annotations for split snippets. In the complementing layer, we devise an inference method that makes use of the indoor topology and the mobility semantics already obtained to recover the missing mobility semantics. The extensive experiments demonstrate that our solution is efficient and effective on both real and synthetic data. For typical queries, our solution's resultant mobility semantics lead to more precise answers but incur less execution time than alternatives.
\end{abstract}

CCS Concepts: • Information systems $\rightarrow$ Mobile information processing systems; Spatial-temporal systems; Data mining;

Additional Key Words and Phrases: Indoor positioning data, semantic trajectory, mobility data mining

ACM Reference format:

Huan Li, Hua Lu, Gang Chen, Ke Chen, Qinkuang Chen, and Lidan Shou. 2020. Toward Translating Raw Indoor Positioning Data into Mobility Semantics. ACM/IMS Trans. Data Sci. 1, 4, Article 26 (November 2020), 37 pages.

https://doi.org/10.1145/3385190

\footnotetext{
This work was supported by the Independent Research Fund Denmark (no. 8022-00366B), the National Science Foundation of China (nos. 61672455 and 61528207), and the Natural Science Foundation of Zhejiang Province of China (no. LY18F020005).

${ }^{*} \mathrm{~L}$. Shou is also with the State Key Laboratory of CAD\&CG, Zhejiang University, China.

Authors' addresses: H. Li and H. Lu (corresponding author), Department of Computer Science, Aalborg University, Denmark; emails: \{lihuan, luhua\}@cs.aau.dk; G. Chen, K. Chen, Q. Chen, and L. Shou, Department of Computer Science, Zhejiang University, China; emails: \{cg, chenk, chenqk, should\}@zju.edu.cn.

Permission to make digital or hard copies of all or part of this work for personal or classroom use is granted without fee provided that copies are not made or distributed for profit or commercial advantage and that copies bear this notice and the full citation on the first page. Copyrights for components of this work owned by others than the author(s) must be honored. Abstracting with credit is permitted. To copy otherwise, or republish, to post on servers or to redistribute to lists, requires prior specific permission and/or a fee. Request permissions from permissions@acm.org.

(c) 2020 Copyright held by the owner/author(s). Publication rights licensed to ACM.

2577-3224/2020/11-ART26 \$15.00

https://doi.org/10.1145/3385190
} 


\section{INTRODUCTION}

According to multiple studies [19, 21], people spend about $90 \%$ of their daily lives indoors. Human movements indoors are increasingly captured in indoor positioning data due to the recent advance in Internet-of-Things (IoT) wireless technologies [1] and high penetration of smartphones [2]. Analyzing indoor positioning data can reveal interesting findings otherwise hard to obtain, such as popular indoor locations [25, 26, 29], hot indoor routes [25, 34], useful indoor patterns [22], and insights for in-store marketing [13].

One class of indoor mobility analysis concerns semantics and asks questions like "Which combination of shops is most frequently visited by the shoppers in a mall?" or "List out the staff that meets at the security-sensitive regions after work hours and stays there for more than an hour." To answer such questions, we need to extract the mobility-related semantics from the underlying indoor positioning data.

In this study, we use a type of data generated by indoor positioning systems based on IoT wireless technology (e.g., Wi-Fi or Beacon) [17, 18]. In particular, the data for one person (or moving object) contains a set of raw positioning records that are uncertain and discrete in nature. Each raw record $((x, y, f), t)$ means an object is observed at coordinate point $(x, y)$ on floor $f$ at time $t$. As the raw data does not give the needed semantics explicitly, we need to translate the raw data into an explicit, informative data representation. Inspired by the semantic trajectories $[16,33,44]$, we use indoor mobility semantics exemplified as follows:

$$
\begin{aligned}
& o_{1}:(\text { stay, Nike, 1:02pm - 1:18pm }) \rightarrow(\text { pass, Adidas, } 1: 19 p m-1: 20 p m) \\
& \rightarrow(\text { stay, Cashier, 1:21pm-1:24pm }) .
\end{aligned}
$$

Specifically, object $o_{1}$ 's movements are represented by a line of structured mobility semantics, which includes a mobility event annotation (a stay or pass event), a spatial annotation (a semantic region like Nike store), and a temporal annotation (a time period). We use $m$-semantics ${ }^{1}$ to refer to such mobility semantics. As the annotations in $\mathrm{m}$-semantics are related to indoor semantic regions and mobility events, m-semantics are considerably more comprehensible and useful for relevant application needs than the raw data [24].

However, translating raw indoor positioning data into m-semantics is still a challenging task mainly due to three reasons. First, the raw data obtained by wireless technology is extremely dirty because of unpredictable interferences of wireless signals $[17,18]$, especially when the infrastructure uses ordinary sensors, such as Wi-Fi access points, for network access [13].

Figure 1 depicts some Wi-Fi based positioning data obtained in a real-world shopping mall in China. Referring to Figure 1(a), for a device sampled every 10s, the average positioning error (APE) calculated every 10 minutes fluctuates significantly. In addition, there are many false floor values reported for two example devices $\mathrm{d} 1$ and $\mathrm{d} 2$ as shown in Figure 1(b). Such data errors must be handled carefully before m-semantics can be extracted. Second, an indoor space usually has a relatively small extent but complex topology, leading to a compact distribution of semantic regions and complex movements under indoor topology. This makes the annotation more difficult. Existing outdoor annotation techniques make use of the POI category [44] or assumptions such as human activity regularity [42]. However, in indoor spaces, POIs of the same type often cluster together but object movements are quite random. Third, indoor positioning data is always discrete as mobile devices tend to turn off wireless signals for energy-saving needs [18]. It is non-trivial to obtain a complete sequence of $\mathrm{m}$-semantics from discrete positioning data that at first glance suggests many possibilities.

\footnotetext{
${ }^{1}$ Here, $m$ stands for "mobility."
} 
(a)

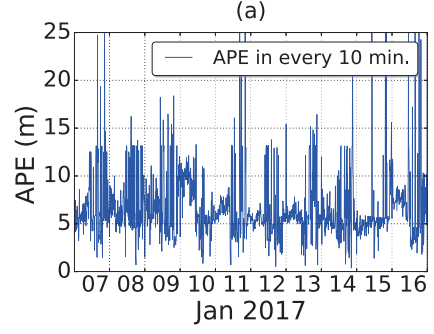

(b)

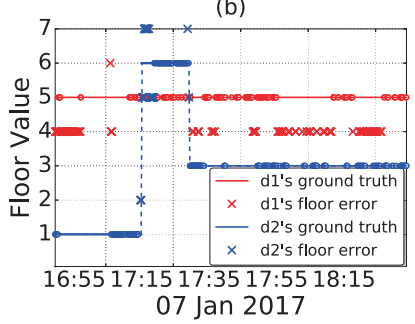

Fig. 1. Real-world indoor positioning data errors.

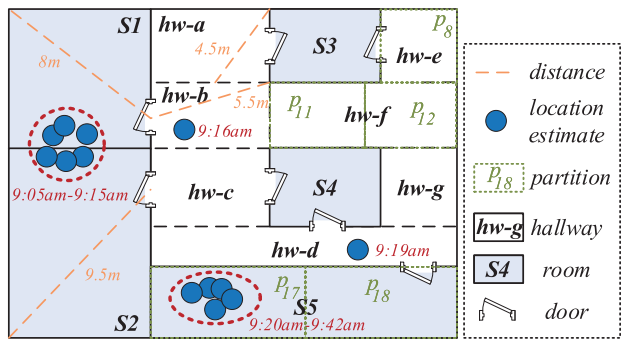

Fig. 2. Example of indoor floorplan.

Example 1. Referring to the floorplan in Figure 2, some semantic regions are pre-defined such as $S 1$ and $h w$-f. We illustrate an object's raw positioning data on the floorplan. Note that the location estimates represented as geometric points $(x, y)$ are barely informative, as no semantics can be recognized without the underlying floorplan provided. When interpreting the positioning data, due to the inherent errors and the layout of indoor regions, it is hard to make the spatial annotation (i.e., semantic region) for the object during the time period 9:05am-9:15am. In addition, it is hard to construct m-semantics between the two presences in hallways $h w-b$ at 9:16am and $h w-d$ at 9:19am, as no straightforward information is available by the discrete indoor positioning records.

To address the aforementioned challenges, we propose a three-layer framework for constructing m-semantics. In the framework, the ultimate task is decomposed and accomplished by three functional layers in a chained manner. Each layer is equipped with corresponding applicable techniques to facilitate data processing. The cleaning layer considers the characteristics of indoor mobility constraints and cleans the raw positioning sequences. Subsequently, in the annotation layer, each positioning sequence is split into a number of snippets according to the underlying mobility event. The snippets are then translated into m-semantics by semantic matching. Last, in the complementing layer, to recover the missing $\mathrm{m}$-semantics in an $\mathrm{m}$-semantics sequence, knowledge about indoor mobility is constructed from the m-semantics already obtained, and an inference based method is used to infer the missing m-semantics on top of the constructed knowledge. Consequently, each object's m-semantics sequence is complemented. A prototype of the framework named TRIPS [24] has been built and released online.

To sum up, we make the following contributions in this article:

- We formulate the problem of translating raw indoor positioning data into m-semantics and propose a three-layer framework to solve the problem (Section 2).

- We design a cleaning method that eliminates indoor positioning data errors based on indoor mobility constraints (Section 3). 
Table 1. Notations

\begin{tabular}{ll}
\hline Symbol & \\
\hline$\theta=(l, t)$ & A positioning record \\
$\Theta_{o}=\left\{\left(l_{1}, t_{1}\right), \ldots,\left(l_{n}, t_{n}\right)\right\}$ & Object o's positioning sequence \\
$r \in R$ & An indoor (semantic) region, a spatial annotation \\
$\tau=\left[t_{s}, t_{e}\right]$ & A temporal annotation \\
$\delta \in\{$ stay,pass $\}$ & An event annotation \\
$\lambda=(r, \tau, \delta)$ & An indoor m-semantics \\
$\Lambda_{o}=\left\langle\lambda_{1}, \ldots, \lambda_{m}\right\rangle$ & Object o's ms-sequence \\
$P T=\left\langle r_{1}, \ldots, r_{m}\right\rangle$ & Region pattern of an ms-sequence \\
$\phi=r_{s} \rightarrow \cdots \rightarrow r_{e}$ & An indoor candidate path \\
dist $_{I}\left(l_{s}, l_{e}\right)$ & Minimum indoor walking distance from location $l_{s}$ to location $l_{e}$ \\
dist $_{g r}\left(r_{s}, r_{e}\right)$ & Guaranteed reaching distance from region $r_{s}$ to region $r_{e}$ \\
\hline
\end{tabular}

Table 2. Example of IPT

\begin{tabular}{cc}
\hline Object & Positioning Record \\
\hline$o_{1}$ & $\left((2.5,10.7,1), t_{1}\right)$ \\
$o_{2}$ & $\left((5.1,38.5,4), t_{1}\right)$ \\
$o_{1}$ & $\left((2.3,11.2,2), t_{4}\right)$ \\
\hline
\end{tabular}

- We devise a split-and-match approach to annotate m-semantics. It includes a density-based method that splits the positioning sequence according to the underlying mobility event and a semantic matching method that makes annotations for the split snippets (Section 4).

- We propose an inference method to recover the missing m-semantics with the help of knowledge about indoor mobility, indoor topology, and m-semantics already obtained (Section 5).

- We conduct extensive experiments on both real and synthetic data to evaluate the efficiency and effectiveness of our proposals (Section 6).

In addition, Section 7 reviews the related work, and Section 8 concludes the article.

\section{PRELIMINARIES}

Table 1 lists the notations used throughout this article.

\subsection{Problem Formulation}

In our setting, an indoor positioning system aperiodically reports a record $\theta(l, t)$ for an object $o$, where $l$ is a location and $t$ is a timestamp, meaning that object $o$ 's location is estimated to be $l$ at time $t$. In most indoor positioning systems $[17,18], \theta . l$ is represented as a triplet $(x, y, f)$-for instance, a $2 \mathrm{D}$ point $(x, y) \in \mathbb{R}^{2}$ on a floor $f \in \mathbb{N}$. The positioning records are stored in an indoor positioning table (IPT), as exemplified in Table 2. Given an IPT and a particular time interval TI, we define an object's indoor positioning sequence (p-sequence) as follows.

Definition 1 (Indoor Positioning Sequence). An object o's indoor positioning sequence over time interval $\mathrm{TI}$ is a time-ordered sequence $\Theta_{o, \mathrm{Tl}}$ of positioning records of $o$, denoted as $\Theta_{o, \mathrm{Tl}}=$ $\left\langle\left(l_{1}, t_{1}\right), \ldots,\left(l_{n}, t_{n}\right)\right\rangle$ such that $\left[t_{1}, t_{n}\right] \subseteq \mathrm{TI}$. 
Referring to Table 2 , the object $o_{1}$ 's p-sequence over $\mathrm{TI}=\left[t_{1}, t_{4}\right]$ is $\left\langle\left((2.5,10.7,1), t_{1}\right)\right.$, $\left.\left((2.3,11.2,2), t_{4}\right)\right\rangle$. We formally give the definition of mobility semantics (m-semantics).

Definition 2 (Mobility Semantics). An object o's mobility semantics is a triplet $\lambda(r, \tau, \delta)$, where the spatial annotation $r$ is an indoor region, the temporal annotation $\tau$ is a time period, and the event annotation $\delta$ is a mobility event.

The indoor regions serving as the spatial annotations are usually pre-defined with particular semantics by data analysts. For example, an indoor region can be a cashier or a shop in a mall. Essentially, an indoor space can be divided into a set of indoor partitions like rooms and hallways by walls and doors. For simplicity, we assume that each indoor region is composed of one or more such indoor partitions. ${ }^{2}$ Referring to Figure 2, a semantic region $h w-e$ corresponds to a single partition $p_{8}$, whereas another semantic region $S 5$ contains two partitions (i.e., $p_{17}$ and $p_{18}$ ).

A mobility event refers to some interesting movement pattern. We introduce two generic patterns in our study, namely stay and pass. ${ }^{3}$ A stay indicates that an object has been staying in a semantic region for a sufficiently long time for a particular purpose that is fulfilled in that region. For example, a stay can be that a user spent half an hour in a shoe shop, selecting and buying a pair of new shoes. It is noteworthy that since an indoor region itself usually carries some rich semantics determined by the use or planning of the space, combining the stay event with the corresponding region is very useful to disclose rich information about user behavior. For example, a stay at a restaurant usually means a person was dining, whereas a stay at an office room indicates that a person was at work. In contrast, a pass tells that an object has passed through an indoor region but there is no particular purpose associated with that pass. For example, a user may pass several other shops before she reaches the shop where she buys shoes. The distinction between stay and pass is useful in pertinent indoor scenarios. For example, security managers may only be interested in people staying in a certain region, whereas the mall managers may want to know both numbers of staying and passing customers when analyzing the customer conversion rate in a region.

As to be introduced in Section 4.2.1, we design an event identification function ( $\mathcal{E}$-function for short) to differentiate a stay and a pass. The $\mathcal{E}$-function is a learning-based model and semisupervised with the spatiotemporal features extracted from the user-recognized mobility events. An m-semantics annotated with a stay (pass) event is referred to as a stay (pass) m-semantics and denoted by $\lambda^{\prime \prime}\left(\lambda^{\triangleright}\right)$, and its associated region is a stay (pass) region denoted as $r^{\prime \prime}\left(r^{\triangleright}\right)$.

Next, we define m-semantics sequence (ms-sequence).

Definition 3 (M-Semantics Sequence). An indoor object o's m-semantics sequence over time interval TI is a time-ordered sequence $\Lambda_{o, \mathrm{Tl}}$ of o's m-semantics: $\forall \lambda_{i}, \lambda_{j} \in \Lambda_{o, \mathrm{Tl}}, \lambda_{i} . \tau \subseteq \mathrm{Tl}, \lambda_{j} . \tau \subseteq \mathrm{TI}$, $\lambda_{i} . \tau \cap \lambda_{j} \cdot \tau=\varnothing$.

When time context is clear, we use $\Theta_{o}$ and $\Lambda_{o}$ to denote object $o$ 's p-sequence and ms-sequence, respectively. We formulate our research problem as follows.

Given an IPT and a full set of indoor semantic regions, the indoor m-semantics construction translates each object $o$ 's p-sequence $\Theta_{o}=\left\langle\theta_{1}, \ldots, \theta_{n}\right\rangle$ in IPT into the most-likely ms-sequence $\Lambda_{o}=\left\langle\lambda_{1}, \ldots, \lambda_{m}\right\rangle$.

Constructing ms-sequences provides an intuitive, concise way to understand a moving object's general indoor behaviors. It serves as the foundation of multiple high-level mobility analyses. However, the major challenge to the construction is that the input data is of very low quality

\footnotetext{
${ }^{2}$ Assuming regions do not overlap, our techniques allow an indoor region to involve (parts of) an indoor partition.

${ }^{3}$ The outdoor patterns that carry similar meanings are known as stop and move in other literature [4, 33, 44].
} 


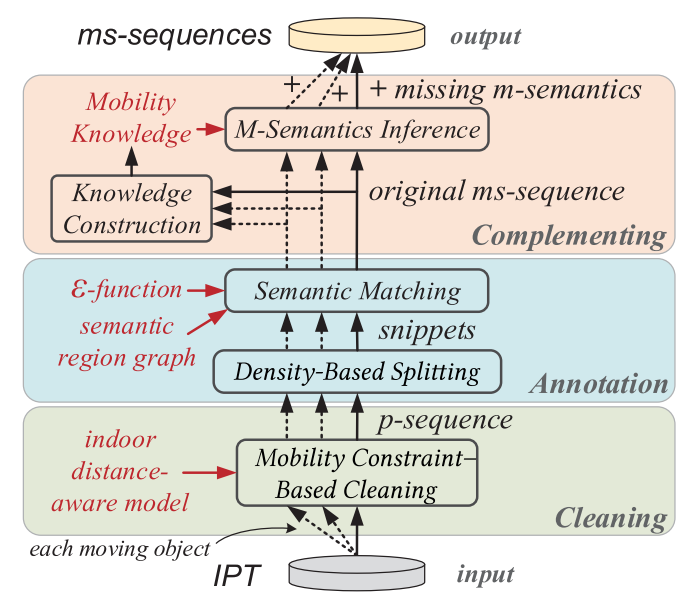

Fig. 3. The construction framework.

and only provides very limited information. To this end, we propose a three-layer framework that progressively improves the data quality and constructs the m-semantics.

\subsection{Framework Overview}

As illustrated in Figure 3, our framework takes each object's p-sequence from the IPT as input and exports the corresponding ms-sequence. The data is processed through three functional layers.

The cleaning layer handles the data errors in the p-sequence of each object. It conducts the data cleaning by considering the indoor mobility constraints captured in a distance-aware model.

The annotation layer first uses a density-based method to split each p-sequence into a number of snippets, then translates each snippet into a number of $\mathrm{m}$-semantics by a semantic matching based on the $\mathcal{E}$-function and the semantic region graph.

The complementing layer recovers the missing $\mathrm{m}$-semantics for each original ms-sequence from the annotation layer. By making use of all of the m-semantics already annotated, the mobility knowledge is obtained by a knowledge construction. Subsequently, each ms-sequence is complemented with a number of missing $\mathrm{m}$-semantics by using an m-semantics inference with mobility knowledge.

The translation framework has been implemented in our prototype system TRIPS [24]. ${ }^{4}$

\section{RAW POSITIONING DATA CLEANING}

As shown in Figure 1, raw indoor positioning data contains inherent errors due to the limitations of the wireless-based indoor positioning [18]. Typical errors are as follows:

(1) Random errors are the small distortions from the true locations. They are caused by the imprecise measurement of wireless signals, which is easily influenced by factors such as temperature, humidity, and window opening or closing [23].

(2) Location outliers are the significant deviations from the true locations. They occur when a mobile client suddenly fails to capture the signals from nearby transmitters. The location outliers discussed here are within the range of a floor.

(3) False floor values are usually seen in multi-floor positioning systems. They occur in a case where a mobile client receives stronger signals from the transmitters on other floors.

\footnotetext{
$\overline{{ }^{4} \mathrm{~A} \text { demo video }}$ and relevant materials are available at https://longaspire.github.io/trips/.
} 


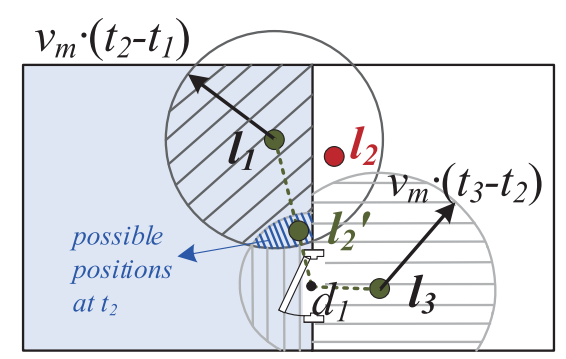

Fig. 4. Example of cleaning raw indoor positioning data.

These data errors impose serious problems on the subsequent processing of indoor $\mathrm{m}$-semantics construction. It is necessary to identify and repair them to reduce their negative impacts.

Generally, indoor object movements should comply with underlying mobility constraints. For example, moving objects (usually people) cannot walk too fast indoors-a significant shift in the positioning data within a short time interval usually means a location outlier or a false floor value. In addition, objects can move between indoor partitions only through doors or the like. Considering the moving speed between two positioning locations under indoor topology, we are able to identify a part of random errors that jump to other indoor partitions. We give an example in Figure 4. Suppose that an object $o$ 's p-sequence is $\left\langle\left(l_{1}, t_{1}\right),\left(l_{2}, t_{2}\right),\left(l_{3}, t_{3}\right)\right\rangle$, and $l_{1}$ at time $t_{1}$ is assumed to be valid. Given the maximum moving speed $v_{m}$ and the specific indoor topology, $o$ 's position at time $t_{2}$ can only be inside the shaded part of the circle centered at $l_{1}$ with a radius $v_{m} \cdot\left(t_{2}-t_{1}\right)$. As o's location report $l_{2}$ is outside the shaded part at time $t_{2}$, the reported location $l_{2}$ is an error.

When computing the object moving speed between two positioning locations, we use the indoor distance-aware model [28] to compute the minimum indoor walking distance (MIWD) between two indoor locations. In Figure 4, the MIWD from location $l_{1}$ to location $l_{3}$, denoted as $\operatorname{dist}_{I}\left(l_{1}, l_{3}\right)$, is computed as the sum of two Euclidean distances $\left|l_{1}, d_{1}\right|$ and $\left|d_{1}, l_{3}\right|$, where $d_{1}$ is the door through which an object can reach $l_{3}$ from $l_{1}$.

Based on the MIWD that integrates both topological and geometrical mobility constraints, we identify the positioning data error by checking the indoor object speed. Formally, given a maximum speed $v_{m}$, for any two consecutive positioning records $\theta_{i}, \theta_{i+1}$, their in-between indoor speed $v=\frac{\operatorname{dist}_{I}\left(\theta_{i} . l, \theta_{i+1} . l\right)}{\theta_{i+1} \cdot t-\theta_{i} \cdot t}$ should not exceed $v_{m}$. In other words, assuming we check a p-sequence in the forward direction, a record $\theta_{i}$ that violates the aforementioned speed constraint should be invalid if the precedent record $\theta_{i-1}$ has already been determined as valid.

When an invalid record $\theta_{i}$ is identified, the error may occur at its floor part (i.e., false floor value) and/or its 2D point part (i.e., location outlier or random error). We repair it in two steps. The first step repairs a potential mistake of floor value. If $\theta_{i}$ 's floor value is different from the previous valid record $\theta_{p}$ 's $(p \leq i-1)$, we modify $\theta_{i}$ 's to the same as $\theta_{p}$ 's. Such modification comes into effect if the violation of speed constraint no longer occurs; otherwise, the potential error is still in the $2 \mathrm{D}$ point part. The second step repairs the wrong location estimate by interpolating a new one: for the current record $\theta_{i}$, we find the previous and next valid positioning record as $\theta_{p}$ and $\theta_{s}(s \geq i+1)$, respectively. As no information is provided about the object movement between the two valid records, we assume that the object moved along the shortest indoor path between locations $\theta_{p} . l$ and $\theta_{s} . l$ at a constant speed. This assumption simplifies the interpolation but still complies with the mobility constraints. Consequently, given the timestamp $\theta_{i} . t$, the corresponding new location estimate is interpolated as a location $l$ on the shortest indoor path from $\theta_{p} . l$ to $\theta_{s} . l$ 
such that $\operatorname{dist}_{I}\left(\theta_{p} . l, l\right)=\frac{\theta_{i} \cdot t-\theta_{p} . t}{\theta_{s} \cdot t-\theta_{p} . t} \cdot \operatorname{dist}_{I}\left(\theta_{p} . l, \theta_{s} . l\right)$. Also referring to Figure 4 , location estimate $l_{2}$ $\left(l_{3}\right)$ is determined as invalid (valid) based on the speed checking. Moreover, we should further repair $l_{2}$ by the second step as the error is in its $2 \mathrm{D}$ point part. Precisely, the possible position at time $t_{2}$ should be inside the blue shaded region provided that $l_{1}$ and $l_{3}$ have been determined as valid. For simplicity, we interpolate the new estimate $l_{2}^{\prime}$ for $o$ at time $t_{2}$ on the shortest indoor path from $l_{1}$ and $l_{3}$ having $\operatorname{dist}_{I}\left(l_{1}, l_{2}^{\prime}\right) / \operatorname{dist}_{I}\left(l_{2}^{\prime}, l_{3}\right)=\left(t_{2}-t_{1}\right) /\left(t_{3}-t_{2}\right)$. It can be proved that $l_{2}^{\prime}$ must be inside the blue shaded region. We omit the details due to the page limit.

The forward version of cleaning is formalized in Algorithm 1. For simplicity of presentation, this version assumes that the first record of the p-sequence is valid. Nevertheless, our cleaning can start with any valid record in the middle such that the remaining ones can be checked forward and backward from the start record. In particular, a record can be selected as the start record if it has both the speeds to its precedent and subsequent records not exceed the threshold $v_{m}$. Similar to the forward cleaning in Algorithm 1, a backward cleaning can be performed to handle the records before the start record selected.

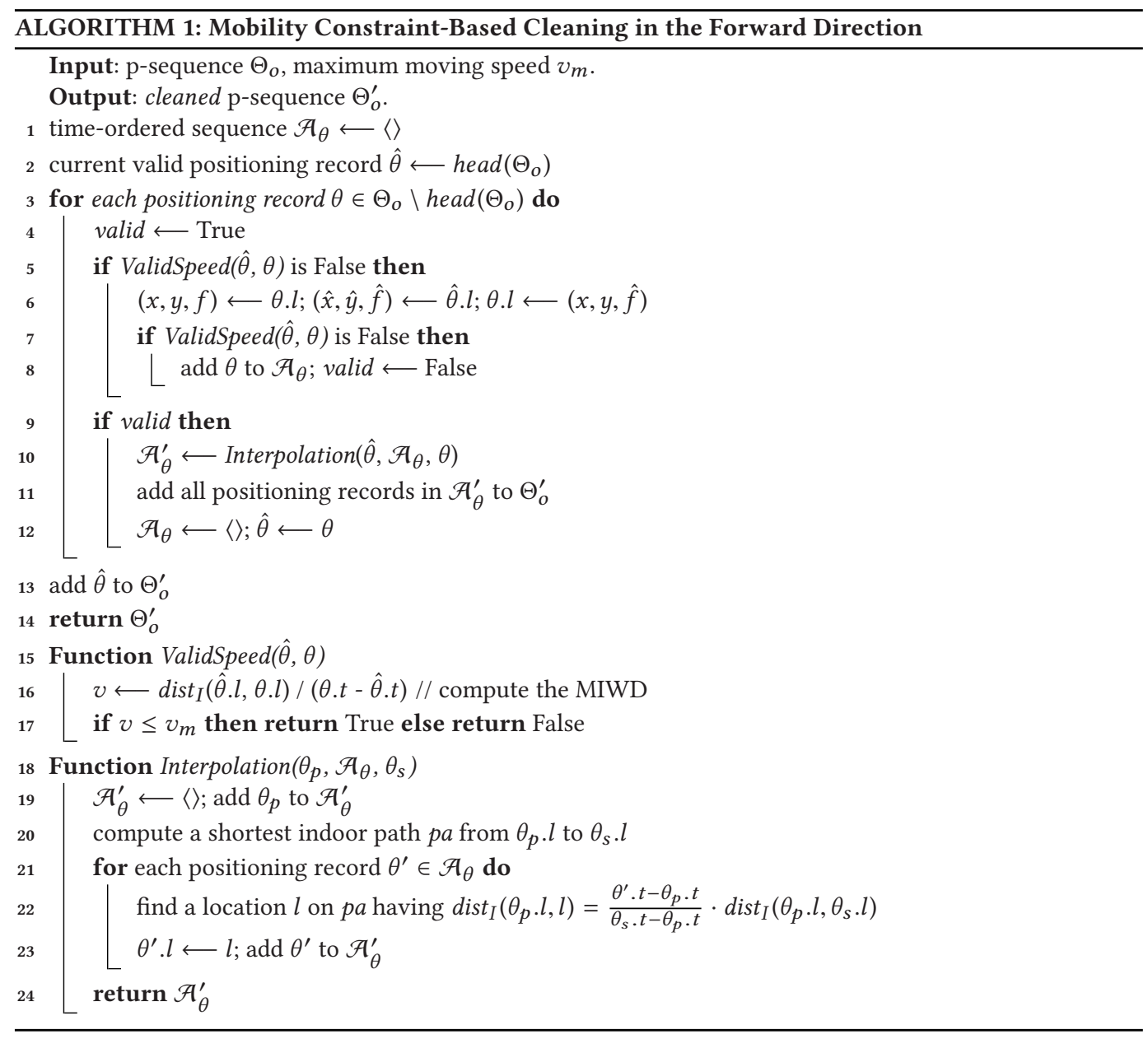




\section{MOBILITY SEMANTICS ANNOTATION}

In this section, we use a split-and-match approach to annotate the m-semantics on a cleaned psequence. First, we split the p-sequence into a number of snippets, each corresponding to an underlying mobility event (i.e., stay or pass introduced in Section 2.1). Next, for each split snippet, we match its m-semantics by making the three annotations (see Definition 2). The overall process is formalized in Algorithm 2. We give the density-based splitting method (called in line 2) in Section 4.1, and elaborate on the semantic matching method (called in line 5) in Section 4.2.

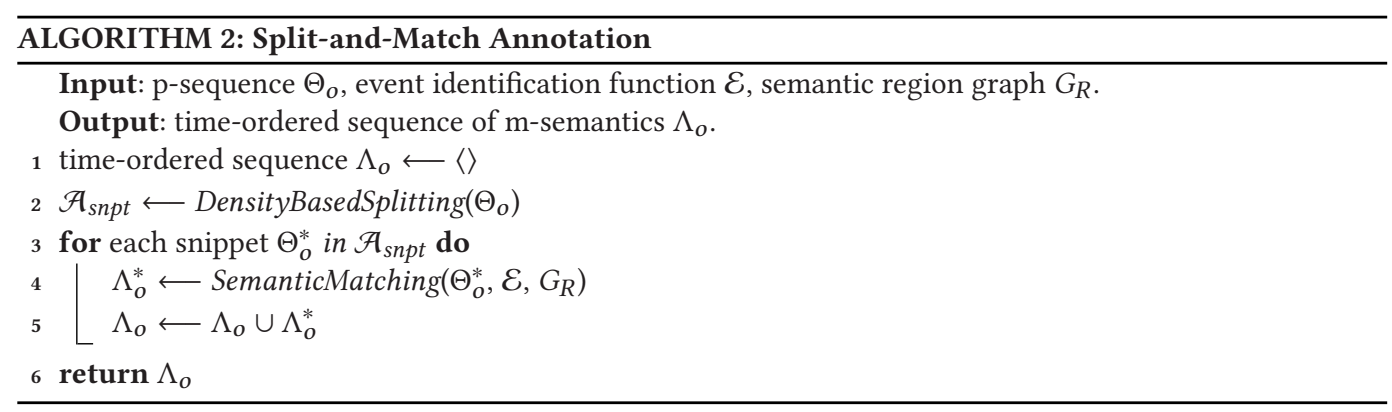

\subsection{Density-Based Splitting}

In our observation, the positioning records associated with a stay event are likely to have their location estimates and timestamps packed together. Inspired by this, we propose a density-based clustering method to find a number of clusters and split the p-sequence based on these clusters. In particular, the positioning records contained in a cluster form a dense snippet, and those consecutive records between two clusters form a non-dense snippet. ST-DBSCAN [8] is a competent algorithm to cluster the sequential data instances according to the spatial and temporal attributes. It requires three parameters: (1) $\epsilon_{s}$ is a distance threshold for spatial attributes, (2) $\epsilon_{t}$ is a distance threshold for temporal attributes; and (3) $p t_{m}$ is a numerical threshold. A cluster is formed only if it contains at least $p t_{m}$ data instances and any instance in it is within the spatial distance $\epsilon_{s}$ and also within the temporal distance $\epsilon_{t}$ to another instance in it. Note that the instances in a formed cluster are continuous on the time attribute. Compared to the traditional speed-based methods like SMoT [4] that use a hard-coded speed threshold to distinguish stay and pass events, our densitybased method takes a more comprehensive consideration of the spatiotemporal data distribution of different mobility events. We experimentally compare different splitting methods in Section 6.1.1.

To enable the density-based splitting on p-sequence, we extend ST-DBSCAN in three aspects. First, we introduce the MIWD as the distance metric for spatial attributes with respect to the indoor topology. Second, we build the parameter $p t_{m}$ adaptively rather than using a constant value. In particular, $p t_{m}$ at time $t_{i}$ is associated with the local sampling rate within the time window $\left[t_{i}-\epsilon_{t}, t_{i}+\epsilon_{t}\right]$. If the local sampling rate is currently low (i.e., only a few positioning records were observed within the time window), we use a small $p t_{m}$. In contrast, a large $p t_{m}$ is used when the local sampling rate is high. This way makes it flexible to form clusters in the context of dynamically changing sampling rates. Third, we introduce two parameters, namely tolerate time span $\Delta_{t}$ and tolerate spatial distance $\Delta_{s}$, to avoid small, fragmentary dense snippets to be formed. Formally, any two dense snippets $\left\langle\theta_{i}, \ldots, \theta_{j}\right\rangle$ and $\left\langle\theta_{k}, \ldots, \theta_{l}\right\rangle$ are merged if (1) $\theta_{k} . t-\theta_{j} . t \leq \Delta_{t}$, and (2) $\exists s \in$ $[i, j], \exists t \in[k, l], \operatorname{dist}_{I}\left(\theta_{s} . l, \theta_{t} . l\right) \leq \Delta_{s}$. The pseudo-codes of the procedure are given in Algorithm 4 in Appendix A.1.

Example 2. Referring to the splitting in Figure 5, the positioning records in a cluster formed within the time period from 9:05am to 9:15am are captured as a dense snippet $D_{1}$. In addition, 


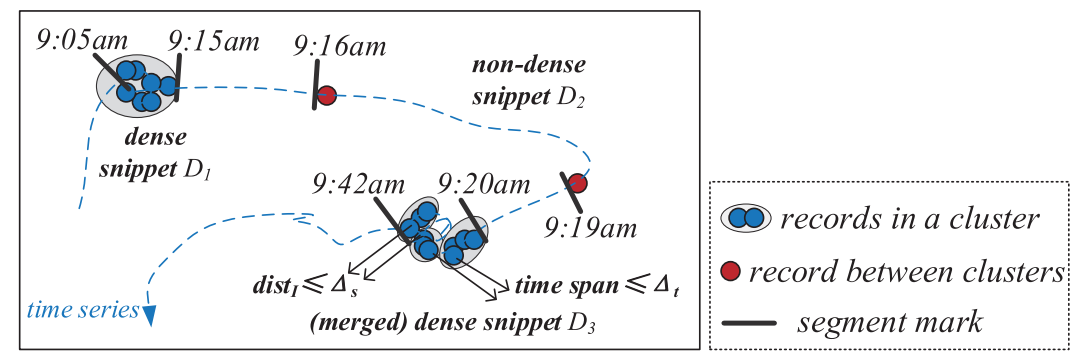

Fig. 5. Example of density-based splitting on a p-sequence.

three captured dense snippets within 9:20am and 9:42am are merged together according to the condition defined on $\Delta_{s}$ and $\Delta_{t}$, resulting in another dense snippet $D_{3}$. Between $D_{1}$ and $D_{3}$, there are two non-clustered records within 9:16am and 9:19am; they form a non-dense snippet $D_{2}$. As a result, the p-sequence is split into three parts.

The density information provides a good reference for splitting a p-sequence. However, it is not sufficient to directly regard a dense snippet as a stay and a non-dense one as a pass. Suppose that an object moves steadily and reports its location at a high frequency. Although the reported locations (and timestamps) can be close to each other and satisfy the clustering conditions, it would be wrong to consider the object is staying in a place. To verify if a snippet corresponds to a true stay event, more information, such as total travel distance and location estimate variance, needs to be extracted from its containing positioning records and be checked further. To this end, we introduce the event identification technique in Section 4.2.1.

\subsection{Semantic Matching}

4.2.1 Event Identification Function. To determine the underlying mobility event associated with a snippet, we design an event identification function ( $\mathcal{E}$-function) based on a semi-supervised learning model. In particular, each snippet (i.e., a segment of raw records) is first represented as a mobility feature vector. Features are extracted from the following aspects:

- Dense level: As the positioning records of stay events usually fall in the formed clusters, it is useful to indicate whether the snippet is dense or not. We represent the dense level of the snippet as a binary value.

- Variance of location estimates: As the variance of location estimates is usually small in stay events but large in pass events, we compute the location variance of the snippet as a feature.

- Sampling conditions: Since the indoor positioning data is usually sparser when the object (wireless device) is moving, we compute the record number and the sampling rate in the snippet as two features.

- Covering range: As a larger covering range tends to be a pass event, we compute the area and the longest distance from the centrum to a vertex of the geometric shape (i.e., convex hull or its simplified minimum boundary rectangle) that covers all location estimates of the snippet.

- Overlapping regions: Considering the effect of different indoor places, we compute the dummy encoded IDs of the neighboring regions that contain or intersect with the covering range, together with each such region's relevant record number. Four nearest regions are considered and the empty dimensions (happens when there are not enough overlapping regions) are filled with zero. 
- Walking distance: As a snippet with a larger walking distance tends to be a pass event, we compute the sum and average of the MIWDs between every two consecutive location estimates as two features.

- Walking speed: As a snippet with a larger walking speed tends to be a pass event, we compute the maximum, minimum, and average of the instant speeds between every two consecutive records as three features.

- Number of turns: Studies reveal that people only make a very small number of turns when they are walking indoors [34]. Therefore, we compute the ratio between the number of turns and the walking distance to help identify pass events. In particular, for any location estimate $\theta_{i} . l$, a turn is identified if the angle between the line from $\theta_{i-1} . l$ to $\theta_{i} . l$ and the line from $\theta_{i} . l$ to $\theta_{i+1} . l$ exceeds 90 degrees.

The logistic regression model [3] is employed to classify stop and pass events. To train the model, a feature set is extracted from the snippets labeled with stop or pass and then normalized. Logistic regression is efficient to train, and it produces a probability value of being one class on the sigmoid curve. Such an advantage enables us to use a co-training method [9] that constructs additional labeled data to overcome the lack of training data. In particular, the predicted snippets with very high or very low probability values can be added as positive and negative samples, respectively.

Consequently, given a snippet $\Theta_{o}^{*}$, its mobility event returned by $\mathcal{E}\left(\Theta_{o}^{*}\right)$ corresponds to either stop or pass predicted by the classifier. Once the mobility event is determined, in Section 4.2.2 we determine the annotations for stay and pass m-semantics, especially the spatial annotation.

4.2.2 Determining Annotations. For a snippet $\Theta_{o}^{*}$ that has $\mathcal{E}\left(\Theta_{o}^{*}\right)=$ stay, a stay $\mathrm{m}$-semantics is generated with the temporal annotation made as $\tau=\left[\right.$ head $\left(\Theta_{o}^{*}\right) . t$, $\left.\operatorname{tail}\left(\Theta_{o}^{*}\right) . t\right]$. In contrast, $\Theta_{o}^{*}$ should be matched to one or more pass m-semantics as the corresponding object may move through different regions. In such a case, each positioning record $\theta \in \Theta_{o}^{*}$ is mapped to a pass m-semantics with its temporal annotation made as $\tau=[\theta . t, \theta . t]$.

Next, we need to make the spatial annotations for the aforementioned stay or pass $\mathrm{m}$-semantics. In the following, we introduce a semantic region graph $G_{R}$ that facilitates accessing the indoor semantic regions. Specifically, $G_{R}$ is a labeled, directed graph represented in a five-tuple $\left(R, E, G_{d i s t}, R 2 P, P 2 R\right)$ :

(1) $R$ is the vertex set, and each vertex in it corresponds to an indoor region $r$.

(2) $E$ is the edge set $\left\{\left\langle r_{i}, r_{j}, \mathbb{R}\right\rangle \mid r_{i}, r_{j} \in R\right\}$, and each directed edge gives the guaranteed reaching distance (GRD) from an indoor region $r_{i}$ to another directly connected ${ }^{5}$ indoor region $r_{j}$.

(3) $G_{d i s t}$ is the associated distance-aware model [28] that involves with indoor entities like doors and partitions.

(4) $R 2 P: R \rightarrow 2^{P}$ maps an indoor region (vertex) to the set of indoor partitions in $G_{d i s t}$ it covers.

(5) $P 2 R: P \rightarrow R$ maps an indoor partition in $G_{\text {dist }}$ to the indoor region that covers it.

The mappings $R 2 P$ and $P 2 R$ are maintained by two hash tables. They can be automatically constructed based on the topological computations between regions and partitions.

\footnotetext{
${ }^{5}$ Two regions are directly connected if an object can move from one to the other without getting into a third region.
} 


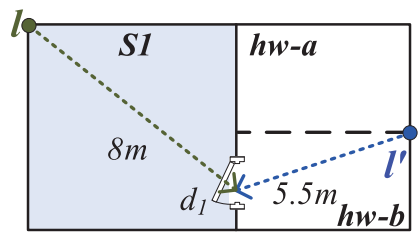

Fig. 6. GRD example.
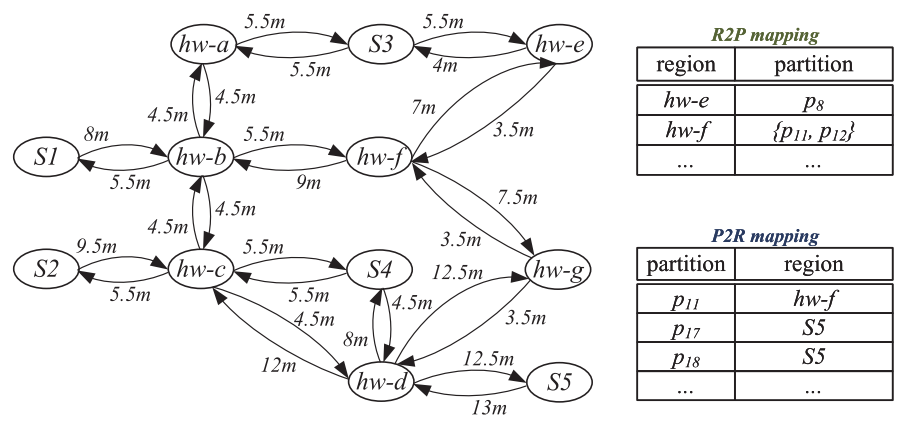

Fig. 7. Example of semantic region graph $G_{R}$.

The GRD from a region $r_{i}$ to a region $r_{j}$ is defined as

$$
\operatorname{dist}_{g r}\left(r_{i}, r_{j}\right)=\max _{l \in r_{i}, d \in P 2 D_{\sqsupset}\left(R 2 P\left(r_{j}\right)\right)} \operatorname{dist}_{I}(l, d),
$$

where $R 2 P\left(r_{j}\right)$ gives the covering partitions of region $r_{j}$ and the mapping $P 2 D_{\sqsupset}$ [28] gives the enterable doors for a given indoor partition. Generally, the GRD from $r_{i}$ to $r_{j}$ is the walking distance an indoor object needs to reach (an enterable door of) $r_{j}$ from the farthest position in $r_{i}$. In other words, any object currently in $r_{i}$ can reach $r_{j}$ within the distance $\operatorname{dist}_{g r}\left(r_{i}, r_{j}\right)$. Note that $\operatorname{dist}_{g r}\left(r_{i}, r_{j}\right) \neq \operatorname{dist}_{g r}\left(r_{j}, r_{i}\right)$. Referring to Figure 6, regions $S 1$ and $h w-b$ are directly connected, whereas $S 1$ and $h w-a$ are not, as their in-between walking path must go through another region $h w-b$. Suppose that $l \in S 1$ is the farthest position from the enterable door $d_{1}$ of $h w-b$. Thus, GRD from $S 1$ to $h w$ - $b$ equals to $\operatorname{dist}_{I}\left(l, d_{1}\right)=8 \mathrm{~m}$. In contrast, GRD from $h w-b$ to $S 1$ is $\operatorname{dist}_{I}\left(l^{\prime}, d_{1}\right)=5.5 \mathrm{~m}$ where $l^{\prime} \in h w-b$ is the farthest position from the enterable door $d_{1}$ of $S 1$. The two GRDs indicate that it usually costs more time to walk out from a larger region like $S 1$ than from a smaller one like $h w-b$. This property of GRDs can be used to allocate the time periods for the regions that have been inferred in recovering the missing $\mathrm{m}$-semantics. The details are to be given in Section 5.2.2.

Figure 7 gives the graph $G_{R}$ corresponding to Figure 2. With the $R 2 P$ and $P 2 R$ mappings, region $h w$ - $f$ is mapped to two partitions that it contains (i.e., partitions $p_{11}$ and $p_{12}$ shown in Figure 2 ) and region $h w-e$ is mapped to a single partition $p_{8}$. In contrast, partition $p_{17}$ in Figure 2 is mapped to its covering region $S 5$. In addition, it can be seen that any object currently in $S 2$ can reach $h w-c$ by a distance $\operatorname{dist}_{g r}(S 2, h w-c)=9.5 m$, consistent with the precise drawing in Figure 2 .

To speed up the spatial searching that involves the geometric location estimates and indoor regions, we index the regions' associated partitions in $G_{\text {dist }}$ by an R-tree. When a given location estimate's intersected partition is found via the R-tree, the relevant covering region can be obtained via the $P 2 R$ mapping defined in $G_{R}$. Note that these mappings allow users to specify the indoor regions without getting to the underlying spatial searching conducted on the level of indoor partitions. Consequently, we can use $G_{R}$ to find the best-matched spatial annotation for an $\mathrm{m}$-semantics according to its corresponding positioning record(s). 
We differentiate the spatial annotation matching for pass and stay $\mathrm{m}$-semantics. For a pass semantics and its corresponding positioning record $\theta$, we simply consider the indoor region that contains $\theta . l$ as the spatial annotation. The consecutive pass $\mathrm{m}$-semantics that are matched with the same spatial annotation should be merged together such that their time periods are combined. This helps reduce possible redundancy in the semantics.

The matching for stay m-semantics is more complex, as it involves multiple location estimates. We find that an object staying in a place usually incurs small displacements between its consecutive location estimates. Therefore, an estimate fairly far away from its neighboring estimates should be affected by positioning errors and is less reliable. In this light, different from the matching methods that use the centroid of location estimates or voting results, we assign each estimate different importance in determining the stay region. We define location estimate confidence as follows.

Definition 4 (Location Estimate Confidence). Given a snippet $\left\langle\theta_{s}, \ldots, \theta_{e}\right\rangle$ associated with a stay event, the confidence of a location estimate $\theta_{i} \cdot l(s \leq i \leq e)$ is defined as

$$
\operatorname{conf}^{(i)}=\left(\frac{\sum_{\theta_{j} \in \mathcal{N}^{(i)}} \operatorname{dist}_{I}\left(\theta_{i} . l, \theta_{j} . l\right)}{\left|\mathcal{N}^{(i)}\right|}\right)^{-1} / Z,
$$

where $\mathcal{N}^{(i)}$ is the set of $\theta_{i}$ 's $k$ nearest neighbor location estimates from other positioning records in the snippet and $Z$ is a normalization parameter making the maximum confidence be 1 .

In the definition, we evaluate each estimate's confidence by making use of its average MIWDs to the neighboring estimates. Enabled by the evaluated confidence, we compute each estimate $\theta_{i} . l$ 's importance as $\beta^{(i)}=\frac{\text { conf }^{(i)}}{\sum_{j=s}^{e} \operatorname{conf} f^{(j)}}$ and infer the underlying stay position as $\hat{l}=\sum_{i=s}^{e} \beta^{(i)} \cdot \theta_{i} . l$. As a result, the spatial annotation for a stay m-semantics is made as a region that contains $\hat{l}$.

The whole procedure of semantic matching is formalized in Algorithm 5 in Appendix A.2.

Example 3. Referring to Figure 5, suppose that the split snippets $D_{1}, D_{2}$, and $D_{3}$ have been determined by the $\mathcal{E}$-function as stay, pass, and stay, respectively. Referring to the snippet $D_{1}$ within 9:05am and 9:15am, its included location estimates are pointed out by a dashed circle in Figure 2. Based on the spatial annotation matching introduced earlier, $D_{1}$ is mapped to an msemantics (S1, 9:05am-9:15am, stay). Similarly, $D_{3}$ within 9:20am and 9:42am is translated into (S5, 9:20am-9:42am, stay) as can be referred in Figure 2. In addition, the two records in $D_{2}$ (within 9:16am-9:19am) is translated into two pass m-semantics-for instance, ( $h w-b, 9: 16 a m-9: 16 a m, ~ p a s s)$ and ( $h w-d, 9: 19 a m-9: 19 a m, p a s s)$.

\section{COMPLEMENTING MS-SEQUENCES}

An ms-sequence obtained from the annotation layer can still be incomplete in that some msemantics are missing between the consecutive pass m-semantics. Next, we present an inference method to recover those missing m-semantics.

Multiple studies [22, 34] have discovered that people often make similar movements between two indoor destinations within a relatively small range, regardless of the walking purposes (e.g., to find something or just to look around somewhere). This inspires us to make use of such similar movements to infer the missing m-semantics between two relevant regions. Specifically, each stay region $r^{\prime \prime}$ in an annotated m-semantics can be considered as an indoor destination, and those annotated pass $\mathrm{m}$-semantics between two destinations can be grouped to capture the similar movements in between. By further considering indoor mobility constraints, we are able to infer the unobserved movements given an ms-sequence we already annotated. 
The complementing consists of two phases, as formalized in Algorithm 3. The first phase (line 2) constructs the mobility knowledge (i.e., similar movements) between each two stay regions (i.e., destinations). Using the constructed knowledge, the second phase (lines 3-5) infers the missing msemantics for each ms-sequence. The two phases are detailed in Sections 5.1 and 5.2, respectively.

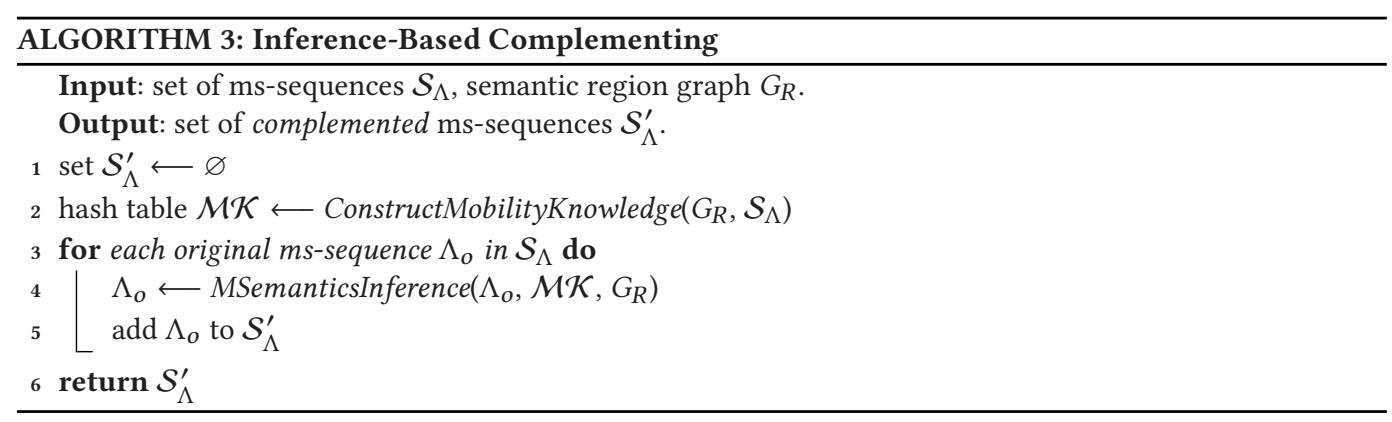

\subsection{Mobility Knowledge Construction}

Given two stay regions $r_{s}^{\prime \prime}, r_{e}^{\prime \prime}$, the mobility knowledge about the similar movements from $r_{s}^{\prime \prime}$ to $r_{e}^{\prime \prime}$ consists of two parts. The first part is a set of candidate paths that accommodate those similar movements, and each path is represented as a sequence of directly connected regions in the semantic region graph $G_{R}$. The second part is the transition probabilities between the directly connected regions in the candidate paths. Next, we elaborate on constructing the candidate path set and the transition probabilities, respectively.

\subsubsection{Candidate Path Set. We first define indoor candidate path.}

Definition 5 (Indoor Candidate Path). Given a start region $r_{s}^{\prime \prime}$ and an end region $r_{e}^{\prime \prime}$, a candidate path from $r_{s}^{\prime \prime}$ to $r_{e}^{\prime \prime}$ is a region sequence $\phi=r_{s}^{\prime \prime} \rightarrow r_{i}^{\triangleright} \rightarrow \cdots \rightarrow r_{j}^{\triangleright} \rightarrow r_{e}^{\prime \prime}$, each pass region $r_{k}^{\triangleright}(i \leq$ $k \leq j$ ) contained in $\phi$ is unique, and each two consecutive regions in $\phi$ are directly connected. Path $\phi$ 's path length $L(\phi)$ is given by

$$
\operatorname{dist}_{g r}\left(r_{s}^{\prime \prime}, r_{i}^{\triangleright}\right)+\sum_{k=i}^{j-1} \operatorname{dist}_{g r}\left(r_{k}^{\triangleright}, r_{k+1}^{\triangleright}\right)+\operatorname{dist}_{g r}\left(r_{j}^{\triangleright}, r_{e}^{\prime \prime}\right),
$$

where dist $_{g r}$ is the GRD kept in $G_{R}$.

The path length $L(\phi)$ is an upper bound of the minimum distance to ensure that any object can reach $r_{e}^{\prime \prime}$ from $r_{s}^{\prime \prime}$ along the path $\phi$. The proof is given in Lemma B.1 in Appendix B.1.

To find a set $\Phi$ of candidate paths from $r_{s}^{\prime \prime}$ to $r_{e}^{\prime \prime}$, we perform an $A^{*}$-Search [48] on $G_{R}$. As the number of such candidate paths can be very large, we use a path length threshold $\gamma$ to filter out the paths whose length is extremely long, as the similar movements are within a relatively small range [22]. The threshold $\gamma$ should be determined according to the statistics on the path lengths between two stay regions. In our experiments, we set $\gamma$ to the double of the length of the shortest candidate path from $r_{s}^{\prime \prime}$ to $r_{e}^{\prime \prime}$, as a path length in the experiments never exceeds this value.

Computing path lengths by using the sum of GRDs between directly connected regions can avoid more complex computations on the underlying distance-aware model $G_{\text {dist }}$. Moreover, it facilitates pruning the irrelevant regions (e.g., those whose sum of GRDs to $r_{s}^{\prime \prime}$ or $r_{e}^{\prime \prime}$ exceeds $\gamma$ ) when searching candidate paths on $G_{R}$.

Example 4. Given the start region $r_{s}^{\prime \prime}=S 1$ and end region $r_{e}^{\prime \prime}=S 5$, the path length threshold $\gamma$ is set to double of $29.5 \mathrm{~m}$, the shortest path length from $S 1$ to $S 5$. As a result, four indoor candidate 
paths can be searched from the $G_{R}$ shown in Figure 7 .

$$
S 1 \stackrel{8 m}{\rightarrow}\left\{\begin{array}{l}
1 . h w-b \stackrel{4.5 m}{\rightarrow} h w-c \stackrel{4.5 m}{\rightarrow} h w-d \\
2 . h w-b \stackrel{4.5 m}{\rightarrow} h w-c \stackrel{5.5 m}{\rightarrow} S 4 \stackrel{4.5 m}{\rightarrow} h w-d \\
3 . h w-b \stackrel{5.5 m}{\rightarrow} h w-f \stackrel{7.5 m}{\rightarrow} h w-g \stackrel{3.5 m}{\rightarrow} h w-d \\
4 . h w-b \stackrel{4.5 m}{\rightarrow} h w-a \stackrel{5.5 m}{\rightarrow} S 3 \stackrel{5.5 m}{\rightarrow} h w-e \stackrel{3.5 m}{\rightarrow} \\
h w-f \stackrel{7.5 m}{\rightarrow} h w-g \stackrel{3.5 m}{\rightarrow} h w-d
\end{array}\right\} \stackrel{12.5 m}{\rightarrow} S 5
$$

5.1.2 Transition Probabilities. Next, we compute the transition probability between each two directly connected regions on a path from the candidate path set. Specifically, given a set of mssequences, a start region $r_{s}^{\prime \prime}$ and an end region $r_{e}^{\prime \prime}$, we first obtain their region patterns through the following steps:

(1) For each ms-sequence, we find all of its subsequence that starts with $\lambda_{s}^{\prime \prime}$ and ends with $\lambda_{e}^{\prime \prime}$, where the m-semantics $\lambda_{s}^{\prime \prime}\left(\lambda_{e}^{\prime \prime}\right)$ corresponds to the region $r_{s}^{\prime \prime}\left(r_{e}^{\prime \prime}\right)$.

(2) For each such subsequence represented as $\left\langle\lambda_{s}^{\prime \prime}, \lambda_{i}^{\triangleright}, \ldots, \lambda_{j}^{\triangleright}, \lambda_{e}^{\prime \prime}\right\rangle$, we obtain its region pattern as $P T=\left\langle r_{i}^{\triangleright}, \ldots, r_{j}^{\triangleright}\right\rangle .^{6}$

We iterate through all ms-sequences and record each obtained region pattern and its count number in a hash table $\mathcal{H}_{P T}$. Subsequently, we compute the transition probability that an object leaves a region $r_{i}$ for a directly connected region $r_{j}$ as follows:

(1) For each region pattern $P T=\left\langle r_{i}, \ldots, r_{j}\right\rangle$ in $\mathcal{H}_{P T}$, we find a subset $\Phi^{\prime}$ of candidate path set $\Phi$, in which all paths hold $P T$.

(2) For each path $\phi \in \Phi^{\prime}$, we compute its path length $L(\phi)$ according to Equation (3). As moving objects tend to choose a shorter path on move [34], each path $\phi$ 's weight $\omega_{\phi}$ among all possible paths is considered to be inversely proportional to $L(\phi)$ and computed as $\omega_{\phi}=L(\phi)^{-1} / \sum_{\phi \in \Phi^{\prime}}\left(L(\phi)^{-1}\right)$.

(3) For each pair of directly connected regions $\left\langle r_{k}, r_{k+1}\right\rangle$ in path $\phi$, its score is incremented by $\phi$ 's weighted score so far. Formally, score $\left(\left\langle r_{k}, r_{k+1}\right\rangle\right)+=P T$.count $* \omega_{\phi}$, where PT.count is $P T$ 's count number in $\mathcal{H}_{P T}$.

(4) After each $P T$ has been processed, we compute the transition probability from $r_{i}$ to $r_{j}$ as

$$
\mathrm{P}_{t}\left(r_{i}, r_{j}\right)=\frac{\operatorname{score}\left(\left\langle r_{i}, r_{j}\right\rangle\right)}{\sum_{r \in \operatorname{Out}\left(r_{i}\right)} \operatorname{score}\left(\left\langle r_{i}, r\right\rangle\right)},
$$

where $\operatorname{Out}\left(r_{i}\right)$ is the set of all directly connected regions in $G_{R}$ that an object can enter after leaving $r_{i}$.

The mobility knowledge construction receives a full set of annotated ms-sequences, and returns a hash table that stores the candidate path set and transition probabilities for each stay region pair. ${ }^{7}$ The detailed algorithm is given in Algorithm 6 in Appendix A.3.

Example 5. Figure 8 gives an example of mobility knowledge construction with respect to Example 4. We organize all candidate paths in a sub-graph of $G_{R}$ for the sake of presentation. For a region pattern $P T=\langle h w-b, h w-c, h w-d\rangle$, two candidate paths $\phi_{1}$ and $\phi_{2}$ that support $P T$ are found,

\footnotetext{
${ }^{6} \mathrm{We}$ omit the start and end regions $r_{s}^{\prime \prime}$ and $r_{e}^{\prime \prime}$ when the context is clear.

${ }^{7}$ In fact, mobility knowledge is only constructed for a small fraction of region pairs, as we set an upper limit to the path length threshold $\gamma$ to constrain the candidate path generation.
} 


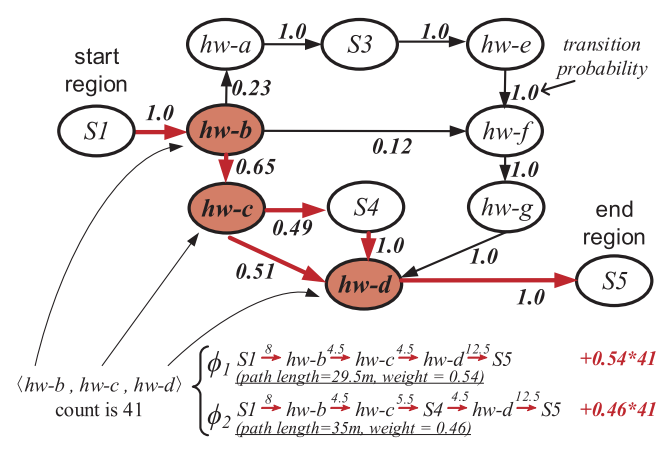

Fig. 8. Example of mobility knowledge construction.

with respective weight of 0.54 and 0.46 . Subsequently, each pair of directly connected regions in $\phi_{1}$ (e.g., $\langle S 1, h w-b\rangle$ and $\langle h w-b, h w-c\rangle$ ) is added with $P T$.count $* 0.54=41 * 0.54$ and each pair in $\phi_{2}$ is added with $41 * 0.46$. After all region patterns in $\mathcal{H}_{P T}$ have been processed, we compute the transition probability for every two directly connected regions. As indicated by the numbers (on the edges) in Figure 8, an object currently seen in $h w-b$ has a probability 0.65 to enter $h w-c$ and only a probability 0.12 to enter $h w-f$.

Note that the mobility knowledge can be updated when batches of $\mathrm{m}$-semantics from the annotation layer are available. We evaluate using such a continuous updating paradigm in Section 6.1.4.

\subsection{Missing M-Semantics Inference}

Given the incomplete observations in an ms-sequence, we infer its missing m-semantics in two steps, namely a most-likely path inference (Section 5.2.1) and a time period inference (Section 5.2.2). The algorithm is formalized as Algorithm 7 in Appendix A.4.

5.2.1 Most-Likely Path Inference. Without loss of generality, we consider a simple problem form: given an observed ms-sequence $\Lambda_{o}^{(o)}=\left\langle\lambda_{s}^{\prime \prime}, \lambda_{q}^{\triangleright}, \lambda_{e}^{\prime \prime}\right\rangle$, our path inference aims to find the mostlikely path that supports its region pattern $P T^{(o)}=\left\langle r_{s}^{\prime \prime}, r_{q}^{\triangleright}, r_{e}^{\prime \prime}\right\rangle$.

Given the candidate path set $\Phi$ constructed for $\left\langle r_{s}^{\prime \prime}, r_{e}^{\prime \prime}\right\rangle$, each path $\phi \in P$ that supports $P T^{(o)}$ can be denoted as $r_{s}^{\prime \prime} \rightarrow r_{a}^{\triangleright} \rightarrow \cdots \rightarrow r_{b}^{\triangleright} \rightarrow r_{q}^{\triangleright} \rightarrow r_{c}^{\triangleright} \rightarrow \cdots \rightarrow r_{d}^{\triangleright} \rightarrow r_{e}^{\prime \prime}$, where $r_{a}^{\triangleright} \rightarrow \cdots \rightarrow r_{b}^{\triangleright}$ and $r_{c}^{\triangleright} \rightarrow \cdots \rightarrow r_{d}^{\triangleright}$ are the missing sub-paths between two consecutive observed regions in $P T^{(o)}$. Note that two consecutive regions in $P T^{(o)}$ are usually not directly connected since the raw indoor positioning records are usually discrete.

Following the literature [30,37] in human mobility prediction, we assume the object movement between regions is a first-order Markov stochastic process-that is, a region an object is currently in is only related to the previous region it went through. Given an observed pattern $P T^{(o)}$, a path $\phi$ 's posterior probability $\mathrm{P}\left(\phi \mid P T^{(o)}\right)^{8}$ satisfies the following expression:

$$
\mathrm{P}\left(\phi \mid P T^{(o)}\right) \propto \mathrm{P}\left(r_{q}^{\triangleright} \mid r_{b}^{\triangleright}\right) \prod_{x=a}^{b-1} \mathrm{P}\left(r_{x+1}^{\triangleright} \mid r_{x}^{\triangleright}\right) \mathrm{P}\left(r_{a}^{\triangleright} \mid r_{s}^{\prime \prime}\right) \cdot \mathrm{P}\left(r_{e}^{\prime \prime} \mid r_{d}^{\triangleright}\right) \prod_{y=c}^{d-1} \mathrm{P}\left(r_{y+1}^{\triangleright} \mid r_{y}^{\triangleright}\right) \mathrm{P}\left(r_{c}^{\triangleright} \mid r_{q}^{\triangleright}\right),
$$

\footnotetext{
${ }^{8}$ Detailed derivation is given in Appendix B.2.
} 
where $\mathrm{P}\left(r_{x+1}^{\triangleright} \mid r_{x}^{\triangleright}\right)$ is equivalent to our captured transition probability $\mathrm{P}_{t}\left(r_{x}^{\triangleright}, r_{x+1}^{\triangleright}\right)$ in Equation (4). To find a most-likely path, we formulate it as a maximum a posteriori problem:

$$
\begin{aligned}
\underset{\phi}{\arg \max }\left(\phi \mid P T^{(o)}\right)= & \underset{\substack{r_{a}^{\triangleright} \rightarrow \cdots \rightarrow r_{b}^{\triangleright} \subseteq \phi \\
\arg \max }}{ } \mathrm{P}_{t}\left(r_{s}^{\prime \prime}, r_{a}^{\triangleright}\right) \prod_{x=a}^{b-1} \mathrm{P}_{t}\left(r_{x}^{\triangleright}, r_{x+1}^{\triangleright}\right) \mathrm{P}_{t}\left(r_{b}^{\triangleright}, r_{q}^{\triangleright}\right) \\
& \underset{\substack{\triangleright \\
r_{c}^{\triangleright} \rightarrow \cdots \rightarrow r_{d}^{\triangleright} \subseteq \phi}}{\arg \max } \mathrm{P}_{t}\left(r_{q}^{\triangleright}, r_{c}^{\triangleright}\right) \prod_{y=c}^{d-1} \mathrm{P}_{t}\left(r_{y}^{\triangleright}, r_{y+1}^{\triangleright}\right) \mathrm{P}_{t}\left(r_{d}^{\triangleright}, r_{e}^{\prime \prime}\right) .
\end{aligned}
$$

The max-product algorithm [15] is used to solve this problem. By taking the transition probabilities from mobility knowledge as input, it finds an optimal sub-path between two consecutive observed regions in the observation $P T^{(o)}$ (e.g., $r_{s}^{\prime \prime}, r_{q}^{\triangleright}$ or $r_{q}^{\triangleright}, r_{e}^{\prime \prime}$ ) as the one with the maximum probability. By assembling the optimal sub-paths it finds, we can obtain a most-likely path for $\Lambda_{o}^{(o)}$.

If no candidate path $\phi$ is found to support the region pattern $P T^{(o)}$, we attribute it to the random errors that jump to another partition. In such a case, we modify $P T^{(o)}$ as follows. For any region $r^{\prime}$ in $P T^{(o)}$ that is not contained by any path in the candidate path set $\Phi$, we change it to the most adjacent region $r^{\prime \prime}$ from those contained by any path in $\Phi$.

Example 6. Referring to the ms-sequence in Example 3, we obtain its observed region pattern $P T^{(o)}=\langle S 1, h w-b, h w-d, S 5\rangle$. To infer the most-likely path, we use the mobility knowledge to find the optimal sub-path between every two consecutive regions in $P T^{(o)}$ (c.f. Equation (6)). Suppose that the optimal sub-paths between $S 1$ and $h w-b, h w-b$ and $h w-d$, and $h w-d$ and $S 5$ are found as $S 1 \rightarrow$ $h w-b, h w-b \rightarrow h w-c \rightarrow h w-d$, and $h w-d \rightarrow S 5$, respectively. The most-likely path is then assembled as $S 1 \rightarrow h w-b \rightarrow h w-c \rightarrow h w-d \rightarrow S 5$.

5.2.2 Time Period Inference. The most-likely path inference finds an optimal sub-path for every two consecutive m-semantics in an observed ms-sequence. Next, we make each region $r_{x} \in \phi^{*}$ a temporal annotation to form the missing m-semantics between $\lambda_{p}$ and $\lambda_{q}$.

For each such region $r_{x}$, its temporal annotation should be divided from the time period between $\lambda_{p}$ and $\lambda_{q}$ (i.e., $\mathrm{MT}_{p, q}=\left[\lambda_{p} . \tau \cdot t_{e}, \lambda_{q} \cdot \tau \cdot t_{s}\right]$ ). However, it is hard to determine the time period that the object is in $r_{x}$ as the object movement is unobserved during $\mathrm{MT}_{p, q}$. In addition, the temporal annotations already made in other observed ms-sequences can hardly be used to infer the time period for $r_{x}$, as the walking speed is variable and different across different objects. To ease computation, we assume that the moving object moves along its path with constant speed during $\mathrm{MT}_{p, q}$. Consequently, we can use the GRD between two regions as the reference ${ }^{9}$ to divide $r_{x}$ 's time period from $\mathrm{MT}_{p, q}$. Formally, for a region $r_{x}$ in $\phi^{*}=r_{p} \rightarrow \cdots \rightarrow r_{q}$, its time period is inferred as $\tau_{x}=\left[t_{s}^{(x)}, t_{e}^{(x)}\right]$, where

$$
t_{s}^{(x)}=\lambda_{p} \cdot \tau \cdot t_{e}+\Delta t \cdot \frac{\sum_{i=p}^{x} \operatorname{dist}_{g r}\left(r_{p}, r_{i}\right)}{\sum_{i=p}^{q} \operatorname{dist}_{g r}\left(r_{p}, r_{i}\right)} ; \quad t_{e}^{(x)}=\lambda_{p} \cdot \tau \cdot t_{e}+\Delta t \cdot \frac{\sum_{i=p}^{x+1} \operatorname{dist}_{g r}\left(r_{p}, r_{i}\right)}{\sum_{i=p}^{q} \operatorname{dist}_{g r}\left(r_{p}, r_{i}\right)}
$$

and $\Delta t=\lambda_{q} \cdot \tau \cdot t_{s}-\lambda_{p} \cdot \tau \cdot t_{e}$ in Equation (7) is the duration of $\mathrm{MT}_{p, q}$.

When the time period $\tau_{x}$ is inferred for $r_{x}$, we differentiate two cases. If $r_{x}$ has already appeared in an observed m-semantics, the time period is added to the corresponding $\mathrm{m}$-semantics. Otherwise, we should generate a missing m-semantics as $\left(r_{x}, \tau_{x}\right.$, pass $)$, which means the object has passed through region $r_{x}$ within the time period $\tau_{x}$. We add each generated m-semantics to the observed ms-sequence and finish the complementing.

\footnotetext{
${ }^{9}$ As discussed in Section 4.2.2, a larger region costs more time to go out as indicated by its GRDs to its connected regions.
} 
Example 7. Continuing with Example 6, we process one of the optimal sub-paths $h w-b \rightarrow$ $h w-c \rightarrow h w-d$. For the regions $h w-b, h w-c$, and $h w-d$ it contains, we divide the time period from 9:16am to 9:19am into three slices according to Equation (7) (i.e., 9:16am-9:18am, 9:18am9:19am, and 9:19am-9:19am). As $h w-b$ and $h w$ - $d$ have appeared in the observed ms-sequence, their time periods are added to the corresponding m-semantics. Moreover, we generate a missing msemantics ( $h w-c$, 9:18-9:19am, pass). Likewise, we process other sub-paths and obtain a complete ms-sequence:

$$
\begin{aligned}
& (\text { S1, 9:05am-9:16am, stay }) \rightarrow(h w-b, 9: 16 a m-9: 18 a m, \text { pass }) \rightarrow(h w-c, 9: 18 a m-9: 19 a m, \text { pass }) \\
& \rightarrow(h w-d, 9: 19 a m-9: 20 a m, \text { pass }) \rightarrow(\text { S5, 9:20am-9:42am, stay })
\end{aligned}
$$

\section{EXPERIMENTAL STUDIES}

All programs are in Java and run on an Intel Xeon E5-2660 2.20-GHz machine with 8 GB of memory.

\subsection{Experiments on Real Data}

Setting. We collected the real data from a Wi-Fi-based positioning system in a seven-floor shopping mall in Hangzhou, China, from January 1 to January 31, 2017. The daily numbers of objects (i.e., device MAC addresses) and positioning records in the operating hours (10am-10pm) were around 7,647 and 2,907,904, respectively. As a result, we obtained a total of 237,057 p-sequences. According to our survey, the positioning data error based on MIWD varied from 2 to $25 \mathrm{~m}$; the average sampling rate was around $1 / 18 \mathrm{~Hz}$ (i.e., a device can be observed about once every $18 \mathrm{~s}$ ). As the indoor partitions divided by walls and doors may be non-convex or imbalanced (long in one dimension but short in the other), we employed an existing decomposition algorithm [43] to divide those irregular partitions into regular ones. As a result, we obtained 3,742 indoor partitions and 6,534 doors. In the mall, 202 shops were selected as semantic regions based on the application needs. The semantic region graph and its associated partition R-tree were kept in memory as they together are only 12.6 MB. The shortest indoor paths between doors were pre-computed to speed up computations on MIWD and GRD. Their maximum memory consumption was $990.8 \mathrm{MB}$.

We used the Event Editor module in TRIPS [24] to visually annotate m-semantics on the p-sequences because we were unable to know a device's exact whereabouts. Among the mssequences we annotated, 1,004 ms-sequences (including 17,322 m-semantics) obtained from January 1 were used to initialize the $\mathcal{E}$-function, and the other 9,687 ms-sequences (including 125,544 m-semantics) from January 2 to January 31 formed the ground truth for evaluation. The purpose of this setting is to study the case when the initial training data is insufficient. In particular, an individual stay m-semantics or a sequence of pass m-semantics formed a snippet, and each snippet was extracted as a 28D feature vector for the model training. From January 2 to January 30, $\mathcal{E}$-function was continuously enhanced by a co-training paradigm [9] in which the most confident predicted snippets (with posterior probability $\geq 0.9$ or $\leq 0.1$ ) were added to the training set at the end of each day. The mobility knowledge was initially constructed for January 1 and also updated daily. The candidate path sets were generated for 10,682 directed region pairs, and the average path set size was 7.7. We kept the mobility knowledge in memory, as it only needs around 36.1 MB.

Performance metrics. In our framework implementation, we used one thread for each object. Therefore, we study the efficiency of our proposed techniques in terms of average running time for processing an individual object's data.

We use two metrics to measure construction effectiveness. On the one hand, we define pointwise accuracy $(\mathrm{PA})$ as the fraction of positioning records that receive correct annotations. As spatial and event annotations are involved for each record, we implement spatial PA (SPA) and event PA 


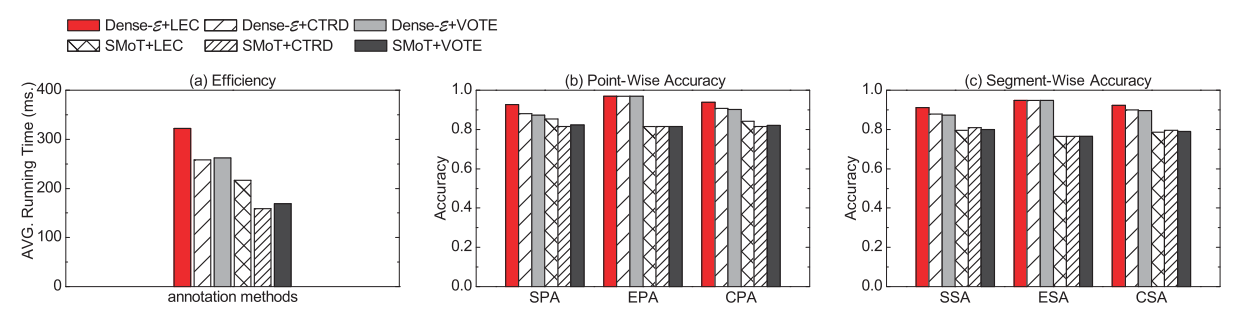

Fig. 9. Performance of annotation methods on real data.

(EPA) that measure PA in terms of spatial annotations and event annotations, respectively. We then define combined PA (CPA) that linearly combines SPA and EPA by a tradeoff parameter $\alpha$ such that $C P A=\alpha \cdot S P A+(1-\alpha) \cdot E P A$. Usually, the SPA requirement is stricter than EPA, as an event annotation makes no sense if the spatial annotation is wrong. On the contrary, analysts can still know a user's whereabouts provided the region is annotated correctly. Therefore, we use a large $\alpha=0.7$ in the evaluation.

On the other hand, we note that sometimes the observed records are distributed unevenly, making some long-period m-semantics not get enough attention in accuracy evaluation. To this end, we segment each constructed ms-sequence at uniform intervals and measure the accuracy of each segment. The metric is called the segment-wise accuracy (SA). Similar to PA, we also implement the spatial SA (SSA), event SA (ESA), and combined SA (CSA). As the sampling rate of real data is relatively low, we set a segment length of 10 minutes. Note that if there is a cross of different m-semantics within a 10-minute interval, we choose the one with a longer duration as the annotations of that segment. The process also applies to the ground truth. In general, PA can be used to assess the accuracy of annotations at a particular time, whereas SA measures the consistency between the annotation and ground truth from a global perspective.

6.1.1 Comparison of Annotation Methods. Our annotation method, denoted as Dense-E+LEC, was implemented as two key modules. One is the temporal-event annotator depending on the density-based splitting method (Section 4.1) and $\mathcal{E}$-function (Section 4.2.1). The other is the spatial annotator that mainly uses the location estimate confidence to match semantic regions for stay m-semantics (Section 4.2.2). We designed several alternatives to compare with Dense- $\mathcal{E}+\mathrm{LEC}$ by modifying its modules. On the one hand, we implemented the SMoT method [4] (described in Section 4.1) for the temporal-event annotator. On the other hand, we used two different matching methods in the counterpart of the spatial annotator. The first called CTRD computes the centroid of all location estimates and selects the covering region as the spatial annotation. The second called VOTE counts the location estimates falling in each region and matches the one with the highest count. By combining these modifications, we obtained five alternative methods: Dense- $\mathcal{E}+C T R D$, Dense-E+VOTE, SMoT+LEC, SMoT+CTRD, and SMoT+VOTE. All alternatives are equipped with a cleaning layer and a complementing layer. Their performances are reported in Figure 9.

Referring to Figure 9(a), all methods based on Dense- $\mathcal{E}$ require more time to annotate msemantics than those with SMoT. Despite the minor time spent in event identification, the time complexity is $O(n \cdot \log n)$ for the density-based splitting and only $O(n)$ for SMoT, where $n$ is the record number of a p-sequence. Comparing the three methods that use the same temporal-event annotator (i.e., Dense- $\mathcal{E}$ or SMoT), the method using LEC costs the most in making spatial annotations because it has to evaluate the confidence for each location estimate. The cost is slightly higher if a method uses VOTE other than CTRD, as VOTE needs to rank all involved regions and select the best. Nevertheless, our Dense- $\mathcal{E}+$ LEC method can process a p-sequence within $350 \mathrm{~ms}$, which is very efficient for most analysis applications. 
Table 3. Effect of Cleaning and Complementing on Real Data

\begin{tabular}{l|c|ccc|ccc}
\hline Method & M-Semantics (\#) & SPA & EPA & CPA & SSA & ESA & CSA \\
\hline IMS-A & 11.94 & 0.8682 & 0.9005 & 0.8779 & 0.3941 & 0.4333 & 0.4059 \\
IMS-CA & 10.23 & $\mathbf{0 . 9 3 9 0}$ & $\mathbf{0 . 9 6 8 4}$ & $\mathbf{0 . 9 2 6 4}$ & 0.4883 & 0.4543 & 0.4781 \\
IMS-AC & 14.51 & 0.8682 & 0.9005 & 0.8779 & 0.7459 & 0.7539 & 0.7483 \\
IMS-CA $\underline{C}$ & 14.12 & $\mathbf{0 . 9 3 9 0}$ & $\mathbf{0 . 9 6 8 4}$ & $\mathbf{0 . 9 2 6 4}$ & $\mathbf{0 . 9 1 2 2}$ & $\mathbf{0 . 9 4 8 7}$ & $\mathbf{0 . 9 2 3 2}$ \\
\hline
\end{tabular}

Figure 9(b) reports the three PAs for all annotation methods. The methods with Dense- $\mathcal{E}$ have the same EPA of 0.968 , which outperforms 0.816 for those with SMoT. This shows that the spatiotemporal density information is more useful than the speed information in splitting p-sequences and identifying mobility events. Looking at SPA, the spatial matching method LEC always outperforms VOTE and CTRD when combining with either Dense- $\mathcal{E}$ or SMoT. CPA performs the best at 0.9264 with the method Dense- $\mathcal{E}+$ LEC. The SAs reported in Figure 9(c) exhibit similar trends with the PAs but are lower in the testing. When a large number of records are observed in a short period, PA tends to give a higher accuracy as each matched record counts one for the hits. In contrast, SA is only processed for each 10-minute segment and thus is less affected by such uneven data distribution. Similarly, Dense- $\mathcal{E}+$ LEC performs the best with the highest SSA, ESA, and CSA at $0.912,0.949$, and 0.923 , respectively.

To sum up, our proposed method Dense- $\mathcal{E}+$ LEC achieves a very good balance between efficiency and effectiveness, and therefore it is very useful in annotating $\mathrm{m}$-semantics.

6.1.2 Effect of Cleaning and Complementing. We compared our complete three-layer framework IMS-CAC (C denotes cleaning and $\underline{C}$ denotes complementing) with several alternatives. Specifically, IMS-A only contains a single annotation layer and two-layer method IMS-CA uses a cleaning layer before annotation, whereas another two-layer IMS-A $\underline{C}$ adds a complementing layer after the annotation layer. Note that the annotation layer is necessary for constructing $\mathrm{m}$ semantics. We report the construction results on the number of $\mathrm{m}$-semantics per ms-sequence, PA, and SA for these four methods in Table 3.

IMS-A is the worst, as it directly annotates m-semantics on the raw data. Its CPA and CSA are 0.8779 and 0.4059 , respectively. Due to the low sampling frequency in the data, SA measures of those methods without data complementing (i.e., IMS-A and IMS-CA) are rather low. In comparison, IMS-CA with a cleaning layer significantly outperforms IMS-A; its CPA is increased at 0.9264 with improvement on both SPA and EPA measures, and all of its SA measures also improve much. Correspondingly, the number of m-semantics per ms-sequence is decreased from 11.94 to 10.23 . These results indicate that our cleaning method repairs many positioning data errors and removes their resultant wrong m-semantics. Therefore, the cleaning layer can improve the input data for subsequent layers. This effect of cleaning is also observed when we compare PA of IMS-CA and IMS-AC. Although the latter produces more m-semantics, many of them are problematic because they result from uncleaned data. Therefore, all PA measures of IMS-A드 are considerably lower than their counterparts of IMS-CA.

IMS-CAC is always the best among all. Both its CPA and CSA are greater than 0.92. Note that the complementing layer takes no effects on PA measures, as the hits are counted only over those observed positioning records. Therefore, PA measures of IMS-CA equal to those of IMS-CAC. However, the SA gap between them is distinguishable. These results show that IMS-CAC can produce reliable $\mathrm{m}$-semantics highly consistent with the ground truth. With the help of our inference-based complementing, IMS-CAC can recover the missing $\mathrm{m}$-semantics that IMS-CA is unable to produce. As a result, the number of m-semantics per ms-sequence is increased from 10.23 to 14.12. More 


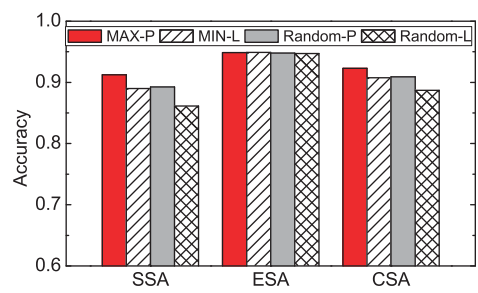

Fig. 10. Complementing comparison.

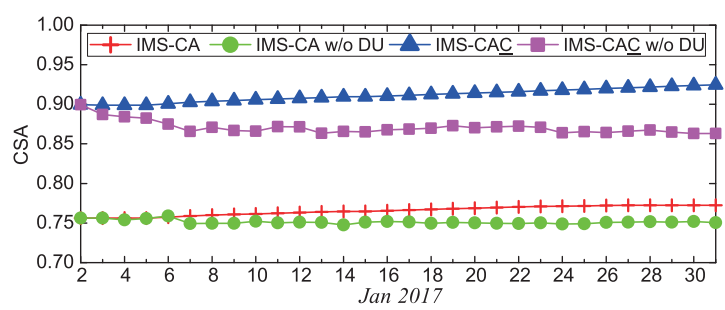

Fig. 11. Effectiveness vs. daily updating on real data.

importantly, because of the combined effects of cleaning and complementing, IMS-CAC's accuracies improve clearly compared to IMS-CA and the others. In summary, the m-semantics construction on real data remarkably benefits from using our complete framework.

We also measure the average running time of processing an object's relevant data for each layer. The time costs in cleaning, annotation, and complementing layers are around 42.6, 321.8, and $11.8 \mathrm{~ms}$, respectively. As the cleaning and complementing layers incur relatively very low time cost while improving the construction effectiveness significantly, it is beneficial and necessary to include them in our framework.

6.1.3 Comparison of Complementing Methods. As existing data repairing techniques [14, 38] are used for data instances and cannot directly support the tuple-form m-semantics, we designed several alternatives to verify the effectiveness of our complementing method. Recall that we used the max-product inference (denoted as MAX-P) in Section 5.2.1 to decode the most-likely indoor path to recover the spatial annotations of the missing $\mathrm{m}$-semantics. We listed several comparable strategies as follows. First, a MIN-L strategy searches for a path with the shortest length between the observed regions as the most-likely path. Different from searching for the optimal path with the shortest length (i.e., MIN-L) or the highest product of transition probabilities (i.e., MAX-P), we can also randomly sample a path among all candidate paths based on their possibilities. The possibility of each candidate path can be measured by either the reciprocal of path length or the product of transition probabilities. The two strategies are denoted as Random- $\mathrm{L}$ and Random- $\mathrm{P}$, respectively. In general, MIN-L and Random-L consider only static space information, whereas MAX-P and Random-P use the transition probability computed upon both space information and historical mobility data. In addition, Random-P and Random-L output stochastic results, whereas the other two give deterministic results. Combining with the time period inference presented in Section 5.2.2, we obtained four complementing methods correspondingly.

With the same cleaning and annotation layers we proposed, we measured the four complementing methods' SAs in the m-semantics construction. Referring to SSAs reported in Figure 10, MAX-P performs the best, whereas the other deterministic method MIN-L is clearly worse. Surprisingly, the stochastic method Random-P also slightly outperforms MIN-L, showing that the transition probabilities are more powerful in capturing the similar movements between regions than the path lengths. Compared to the path length, our transition probability considers both space information and historical object movements. In addition, Random- $L$ is the worst of all. However, ESA changes very slightly as the path inference methods have an impact on the boundary values. In general, our complementing based on the captured mobility knowledge is effective for the missing data recovery. Note that the preceding methods all select the most-likely path from a candidate set found by the $\mathrm{A}^{*}$ algorithm. In this sense, their running time costs are very close.

6.1.4 Effect of Daily Updating Paradigm. We also study the effect of using a daily updating paradigm in constructing $\mathrm{m}$-semantics. In the daily updating case, $\mathrm{m}$-semantics annotated from 
the previous day were accumulated for re-training $\mathcal{E}$-function and updating mobility knowledge. In the case without daily updating, $\mathcal{E}$-function and mobility knowledge were only built with the data obtained from January 1 . We measured CSA for the IMS-CA and IMS-CAC methods on each day from January 2 to January 31. The methods without daily updating are marked with "w/o DU." Referring to Figure 11, the measures of the methods without daily updating fluctuate a lot and in general decrease as the day goes on, whereas those of the methods with daily updating improve and stabilize as time goes by. When the daily updating paradigm is employed, $\mathcal{E}$-function and mobility knowledge are continuously enhanced by more annotated $\mathrm{m}$-semantics. Note that IMS-CAC increases more rapidly than IMS-CA, as it exploits an additional complementing layer where the mobility knowledge can be periodically updated. The results show it is very helpful to update $\mathcal{E}$-function and mobility knowledge when raw positioning data is continuously streamed in.

6.1.5 M-Semantics' Ability to Answer Queries. We also evaluated our constructed m-semantics' ability to answer typical queries. Given a query set $Q$ of indoor semantic regions and a query time interval QT, we introduce two top- $k$ queries:

(1) A Top-k Popular Region Query (TkPRQ) finds $k$ semantic regions from $Q$ that have the most number of visits ${ }^{10}$ within QT.

(2) A Top- $k$ Frequent Region Pair Query (TkFRPQ) finds $k$ most frequent pairs of semantic regions from $2^{Q}$ that both have been visited by the same object within QT.

TkPRQ and TkFRPQ are very useful in studies like popular indoor location discovery $[25,26]$ and frequent pattern mining [22]. Other than m-semantics constructed by the four methods in Table 3, we use the corresponding raw data and cleaned raw data to answer the two queries. The two corresponding methods are denoted as $R A W$ and $R A W-C$, respectively. We introduce a naive strategy to each method-for instance, we compute the visits for all query regions (or region pairs) and return the top- $k$ results by a full ranking. As no semantics is provided in the raw positioning data, the interpretation of $a$ visit to a semantic region is done for the RAW and RAW-C methods. In particular, if an object's reported locations have been falling in a region over a time interval, the object is considered to have visited that region for once. We set the corresponding time interval to 1.5 minutes in RAW and 3 minutes in RAW-C for an optimized tuning.

We compared all methods' efficiency in terms of query execution time. In addition, we evaluated their effectiveness with respect to the ground truth results computed from the ground truth msemantics described in the experimental setting. In particular, we used the metric precision that measures the ratio of the true top- $k$ regions (or region pairs) in the returned top- $k$ results. We issued 20 random queries for each query type and report the average efficiency and effectiveness measures. We fixed $k=60$ and randomly pick 101 (50\% of all) semantic regions to the query set $Q$. We varied the query time interval QT as 60,120, 180, and 240 minutes.

The efficiency results for TkPRQ and TkFRPQ are reported in Figure 12(a) and (b), respectively. In each test, the four IMS methods are faster than RAW and RAW-C by almost two orders of magnitude. When QT increases, more data (positioning records in RAW/RAW-C and m-semantics in IMS methods) should be loaded, and both queries incur more time to return the results. As the scale of raw data is much greater than that of m-semantics, RAW's and RAW-C's execution time increases more rapidly than all IMS methods. In fact, raw positioning data collected in a month was around 3.44 GB, whereas m-semantics constructed by IMS-CAC was only $220.1 \mathrm{MB}$. Moreover, IMS-CAC can return the results for both TkPRQ and TkFRPQ within $1 s$ for a 4-hour

\footnotetext{
${ }^{10}$ In the query context, a visit is equivalent to a stay event.
} 


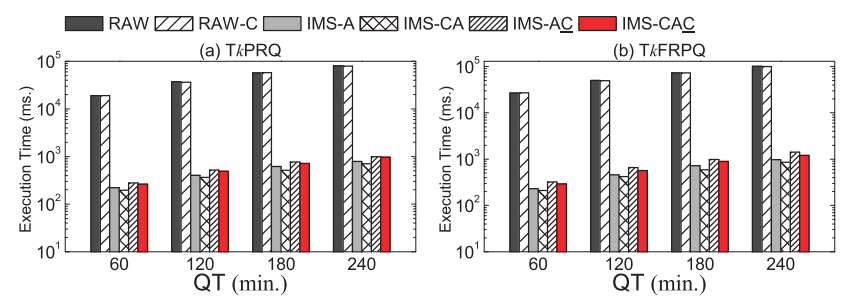

Fig. 12. Query answering efficiency vs. QT on real data.

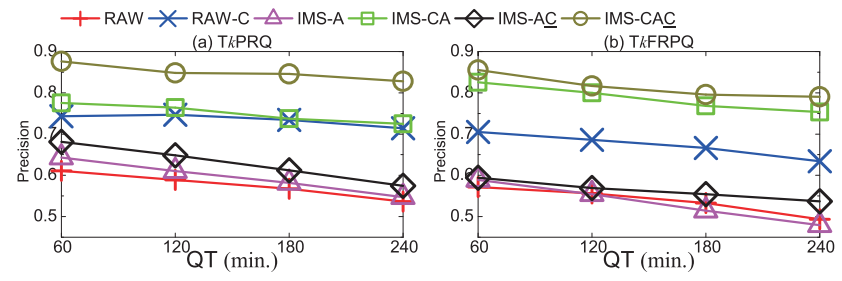

Fig. 13. Query answering effectiveness vs. QT on real data.

query. These results verify that our constructed m-semantics are very efficient in answering the two top- $k$ queries.

We also measured the effectiveness in each aforementioned setting. As shown in Figure 13(a) and (b), for both types of queries, the precision of all methods decreases with an increasing QT. When a longer QT is used, more relevant data should be considered in the query processing, which involves more data errors and makes the results less effective. Nevertheless, all methods with cleaning (i.e., RAW-C, IMS-CA, and IMS-CAC) decrease very slowly. Among them, IMS-CA and IMS-CAC always outperform RAW-C, showing that the brief information kept in m-semantics can capture the underlying object movements very well. Moreover, when QT increases to 240 minutes, m-semantics constructed by IMS-CAC can still achieve a precision $82.8 \%$ for T $k$ PRQ and $79.1 \%$ for TkFRPQ.

We also studied the effectiveness by varying other parameters. In general, the precision results on varying $k$ and $|Q|$ also verify that the m-semantics constructed by IMS-CAC are highly effective in answering both queries than other methods. Such additional results can be found in Appendix C.1.

\subsection{Experiments on Synthetic Data}

We used synthetic data to further verify our proposals' effectiveness when different levels of temporal sparsity and positioning errors are present in the raw indoor positioning data.

By using the indoor mobility data generator Vita [23], we simulated a 10-floor building environment with four staircases, 1,410 indoor partitions, and 2,200 doors. A total of 423 semantic regions were decided upon the partitions at random. We generated moving objects in the environment for 4 hours. Specifically, $10 \mathrm{~K}$ objects were distributed to the floors, each having a lifespan varied from $10 \mathrm{~s}$ to 4 hours. Object maximum speed was set to $V_{\max }=1.7 \mathrm{~m} / \mathrm{s}$ and object movements followed the waypoint model [20]. In particular, each semantic region is considered as a destination, an object moves toward its destination along a pre-planned indoor path, it stays at the destination for a random period from $1 s$ to 30 minutes after arrival, and it moves again to the next destination that is decided randomly. We recorded an object's location every second as the ground truth trajectory, and generated its true m-semantics according to the simulated behavior-for instance, staying at 
Table 4. Synthetic IPT Instances

\begin{tabular}{c|c|c}
\hline IPT Instance & Parameter Setting & Generated Records (\#) \\
\hline$T 5 \mu 3$ & $T=5 s, \mu=3 m$ & $15,231,971$ \\
$T 5 \mu 4$ & $T=5 s, \mu=4 m$ & $15,230,508$ \\
$T 5 \mu 5$ & $T=5 s, \mu=5 m$ & $15,218,742$ \\
$T 10 \mu 3$ & $T=10 s, \mu=3 m$ & $7,416,906$ \\
$T 15 \mu 3$ & $T=15 s, \mu=3 m$ & $4,945,824$ \\
\hline
\end{tabular}
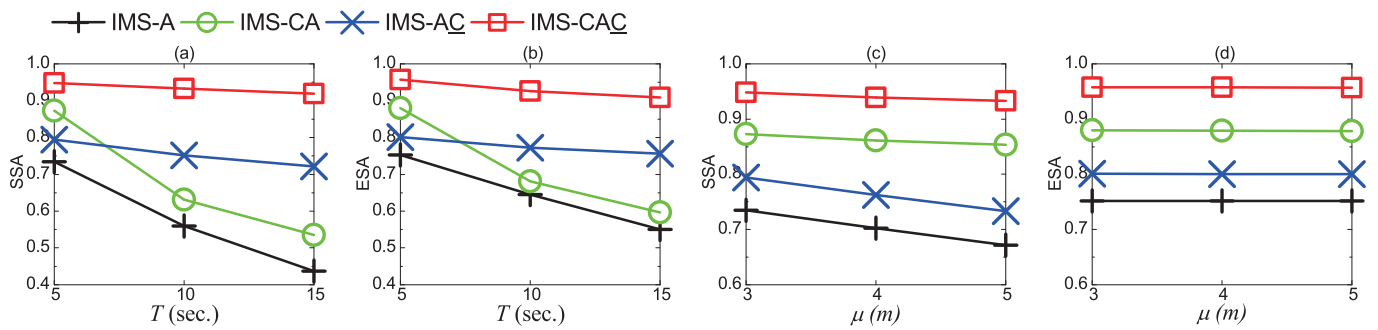

Fig. 14. Effectiveness of m-semantics construction on synthetic data.

(moving toward) a destination was regarded as a stay (pass) event. We obtained 998,618 ground truth m-semantics from the $10 \mathrm{~K}$ objects' ms-sequences.

The synthetic IPT is maintained according to the ground truth trajectories as follows. After an object has sent an update to IPT, it keeps silent for at most $T$ seconds. The maximum positioning period $T$ refers to the maximum value of the time interval between two consecutive positioning records of an object. A location update is randomly within $\mu$ meters from the true location. False floor values and location outliers are added to the updates with certain probabilities (3\% and 3\%, respectively). In particular, a false floor value is produced within two floors up or down, and an outlier is randomly within $2.5 \mu-10 \mu$ meters from the true location. The positioning error factor $\mu$ measures the average distance between the positioning location and its true location. To test the effects of temporal sparsity and positioning error, we varied $T$ and $\mu$, respectively. The synthesized IPT instances are listed in Table 4.

The memory consumptions for $G_{R}$, shortest indoor paths between doors, and mobility knowledge were 13.6, 458, and $48 \mathrm{MB}$, respectively. We randomly selected $3 \%$ of the ground truth mssequences to train $\mathcal{E}$-function, and the rest (including $968,660 \mathrm{~m}$-semantics) were used as testing data in the evaluation.

$M$-semantics construction effectiveness. We tested the four methods in Table 3 on different IPT instances. Here we use SA with a segment length of 10 minutes to measure the consistency between constructed $\mathrm{m}$-semantics and the ground truth described earlier. First, we fixed $\mu=3 \mathrm{~m}$ and vary $T$. SSA and ESA are reported in Figure 14(a) and (b), respectively. When varying $T$ from $5 s$ to $15 s$, for instance, the observed data becomes sparser (Table 4), and all methods' two SA measures decrease but IMS-CAC's decreases the slowest. In addition, the performance gap between IMS-CAC and IMS-CA tends to expand when a larger $T$ is involved, showing that our data complementing is very effective at recovering the missing $\mathrm{m}$-semantics when the positioning data becomes sparser. When $T=15$ s, IMS-CAC can still have an SSA of 0.91 and an ESA of 0.90 . Still, IMS-A's accuracy is the worst and decreases rapidly when $T$ increases. IMS-CA and IMS-CAC equipped with a cleaning layer clearly outperform the other two in all tests. 


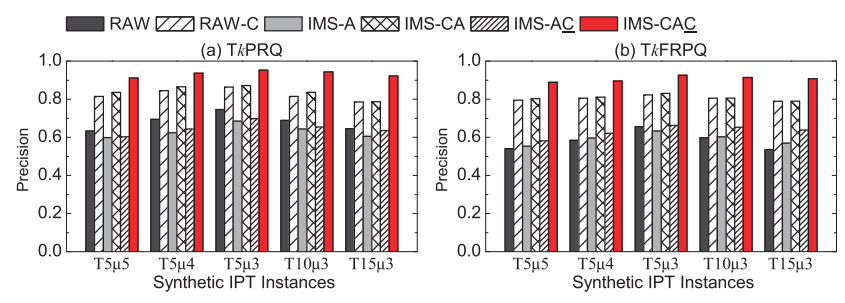

Fig. 15. Query answering effectiveness on synthetic data.

We also fixed $T$ to $5 s$ and tested with different positioning error factors. Refer to the two SA measures reported in Figure 14(c) and (d). When the positioning error factor $\mu$ increases, SSA of both IMS-CAC and IMS-CA stay stable, whereas the others without cleaning decrease rapidly. This demonstrates that our raw data cleaning is very useful to reduce the negative impact of positioning errors. In addition, IMS-CAC outperforms IMS-CA in all tests due to the benefits of the complementing. However, the ESA of all methods is insensitive to $\mu$ and our method's ESA can still be higher than 0.95 .

The results reported in different $T$ and $\mu$ values show that the IMS-CAC framework works very effectively at constructing $\mathrm{m}$-semantics even when the raw data quality is relatively low.

With the ground truth trajectories in synthetic data, we also studied the effectiveness of our raw data cleaning method alone. The additional results in Appendix C.2 demonstrate that our raw data cleaning method is still effective for different $T$ and $\mu$ values.

$M$-semantics' ability to answer queries. For each IPT instance, we answered the TkPRQ and TkFRPQ queries with the six methods introduced in Section 6.1.5. In the experiments, the time interval that indicates a visit to a region was tuned to 2 minutes in RAW and 3.5 minutes in RAWC. A total of 212 (50\% of all) semantic regions were picked to form query set $Q, k$ was set to 60 , and QT to 120 minutes. The precisions of different methods are reported in Figure 15.

Referring to the results of $T 5 \mu 5, T 5 \mu 4$, and $T 5 \mu 3$ in Figure 15(a) and (b), precisions of all methods decrease when $\mu$ increases in both queries. All methods with data cleaning outperform the others in the tests. When $\mu$ increases to $5 m$, our proposed IMS-CAC can still have a precision of $91.06 \%$ for TkPRQ and $88.84 \%$ for TkFRPQ, showing very high effectiveness in answering the two queries when the raw data contains many errors.

Referring to the results of $T 5 \mu 3, T 10 \mu 3$, and $T 15 \mu 3$ for the two queries in Figure 15(a) and (b), the precision of each method decreases with an increasing T. However, IMS-CAC using the complementing decreases very slightly, whereas RAW-C and IMS-CA deteriorate more rapidly. These results show that our complementing is very useful to improve the constructed m-semantics, especially when the raw data is temporally sparse. IMS-A performs very poorly for both queriessometimes it is even worse than directly using the raw data. Thus, it is very necessary to employ all three layers in constructing reliable $\mathrm{m}$-semantics for query use.

\section{RELATED WORK}

Semantic trajectory representation. Parent et al. [33] propose the concept of semantic trajectory as a (GPS) data trace enhanced with annotations and/or complementary segmentations. Güting et al. [16] generalize this concept to symbolic trajectory, a sequence of pairs of a time interval, and a label that refers to some particular user semantics. Marketos et al. [31] design a trajectory reconstruction method to transform raw trajectories into key movement features needed by specific warehousing applications. Zheng and Xie [47] describe a trajectory only by some stay points where the moving objects stop for a relatively long time. Su et al. [39] propose a partition-andsummarization approach, in which a raw trajectory is segmented according to moving object's 
behavior, and the characteristics of each trajectory segment are summarized by a short text. Nogueira et al. [32] propose a framework with ontology to enrich GPS traces with Linked Open Data (LOD). Compared to these works, the $\mathrm{m}$-semantics proposed in this study provide a structured representation about where-when-what of indoor users, which facilitates mobility analytics applications like semantic location prediction [37] and activity recommendation [47].

Semantic annotation. Zhang et al. [46] derive fined-grained sequential patterns by a top-down splitting of the coarse-grained patterns obtained from POI grouping. Alvares et al. [4] extract stop and move events from trajectory points based on geographical information. Cao et al. [10] propose techniques for extracting from GPS data semantically meaningful geographical locations visited by users. Teng et al. [36] identify indoor stop-by pattern as a sequence of occurrence regions from uncertain RFID data. Different from the sequential patterns [46] and visiting location patterns [4, 10, 36], our work annotates two generic mobility patterns-stay and pass, which are flexible for analyzing user behaviors by combining relevant information from indoor regions.

Liao et al. [27] extract activity types and significant places from a person's GPS traces using a hierarchical CRF. Yan et al. [44] propose an HMM-based annotation method to infer stops and POI category for raw GPS records. Wu et al. [42] annotate location records with keywords extracted from geo-referenced social media data by kernel density estimation. By analyzing spatiotemporal regularity, $\mathrm{Wu}$ and $\mathrm{Li}$ [41] study the personalized annotation that enriches personal GPS records with the POI category. Our work differs from these works in several aspects. First, our work searches for a particular region as spatial annotation, whereas other works focus on inferring textual [42] or categorical [27, 41, 44] information for location records. Second, our work uses the spatiotemporal characteristics of a positioning sequence under indoor topology to make annotations, whereas other works demand additional knowledge in geographic spaces, such as human activity regularity [27, 41] and POI category [44]. However, such priors are difficult to meet in indoor spaces with relatively small extents but complex topology. Third, our work makes use of historical mobility knowledge to recover the missing annotations, whereas none of these studies consider the data complementing after annotation. Unfortunately, the data sparsity in a compact indoor space poses even greater challenges than the wider-range geographical space.

Mobility data cleaning and repairing. Mobility data can be in the form of a sequence of geometric locations (e.g., GPS or Wi-Fi positioning data) or symbolic locations (e.g., RFID reader or Bluetooth hotspot). Smoothing-based methods $[14,38]$ are commonly used for handling noisy geometric locations by mathematically interpolating the points on the time series. In contrast, constraintbased methods use additional information such as route structure [35] or other sensory data [12] to mitigate the positioning error. Differently, our cleaning method utilizes the indoor topology that is neither embedded in the smoothing-based methods [14, 38] nor considered by the existing constraint-based methods [12,35]. The interpolation methods $[14,38]$ require inputs captured as points, and thus such methods fall short in complementing $\mathrm{m}$-semantics for which the inputs are regions and three-tuple events.

Similar to the most-likely path inference in our m-semantics complementing, Zheng et al. [45] and Wei et al. [40] study the possible route inference for road networks and free spaces, respectively. However, these techniques are different from our proposal. First, the routable graph built in the work of Wei et al. [40] regards that each two adjacent geospatial grids are connected, unable to model the topological constraints dominated in indoor spaces. Second, the graph-based inference and the nearest neighbor-based inference in the work of Zheng et al. [45] models transition probabilities using only the frequency of relevant historical trajectories. In contrast, region transition probabilities defined in our study consider both movement pattern frequency and distance weights between regions. In Section 6.1.3, we demonstrated that the mobility knowledge that combines both movement and distance information significantly enhances our path inference. 
Using indoor topology to clean and/or repair symbolic location sequences has been studied. Chen et al. [11] clean RFID readings by a Bayesian inference approach that makes use of duplicate RFID readings and prior knowledge about the environment and readers. Baba et al. clean RFID tracking data by utilizing either the integrity constraints [6, 7] implied by RFID reader deployment or the relevant knowledge [5] learned from historical data. In their early solutions, distance-aware graph [7] and probabilistic graph [6] are designed to handle the false positives and false negatives, respectively. Their learning-based approach [5] uses an indoor RFID multi-variate HMM to build the correlation of indoor object locations and RFID readings to handle both false positives and false negatives. Compared to the RFID data captured as discrete states at different timestamps, the Wi-Fi positioning locations in our setting are continuous coordinates that can hardly be optimized with unlimited possible values. In addition, our cleaning method identifies and repairs three types of data errors in one pass, whereas that of Baba et al. [6, 7] can only handle one specific type of data error in a separate and specialized process. Moreover, our inference method for recovering missing data is different from the state detection model [11], probabilistic graph [6], and multi-variate HMM [5] in three aspects. First, our inference is based on the mobility constraints captured at the m-semantics level. Second, we model the similar movements between each pair of semantic regions, whereas Baba et al. [5, 6] and Chen et al. [11] build prior knowledge in a global scope. Third, unlike other works, our transition probability computation considers the effect of the corresponding path's walking length.

\section{CONCLUSION AND FUTURE WORK}

This article tackles the problem of translating mobility semantics from raw indoor positioning data. We propose a three-layer framework with a set of novel techniques. In the cleaning layer, we design a mobility constraint-based cleaning method that eliminates indoor positioning data errors. In the annotation layer, we design a density-based method to split the cleaned data sequence into snippets according to the spatiotemporal densities of the data, and a semantic matching method to make proper annotations and decide mobility semantics for the snippets. In the complementing layer, we devise an inference method to recover the missing mobility semantics with the mobility knowledge captured from historical data. The experiments on real and synthetic data verify that our framework is efficient and effective in constructing mobility semantics, and the constructed mobility semantics are able to answer typical queries efficiently and effectively.

For future work, it will be useful to incorporate the temporal annotations of mobility semantics in inferring the missing data. It also will be interesting to model and learn the probabilistic dependencies among the attributes of mobility semantics to annotate the most-likely semantic regions and mobility events for the positioning sequences. In addition, it will be useful to enrich the mobility semantics by making use of other user behavior data such as transaction logs or check-ins.

\section{APPENDICES}

\section{A ALGORITHMS}

\section{A.1 Density-Based Splitting Algorithm}

Algorithm 4 divides a cleaned p-sequence $\Theta_{o}$ into a sequence of data snippets. It mainly consists of a procedure of density-based clustering (lines 2-19) and a procedure of p-sequence partitioning (lines 20-33).

First, a time-ordered sequence $\mathcal{A}_{\text {snpt }}$ is initialized to hold the split snippets (line 1). Subsequently, the density-based clustering is conducted on the positioning records in the p-sequence $\Theta_{o}$ (lines 219). In particular, the function RetrieveNeighbors (lines 5 and 17) issues a range query on $\Theta_{o}$ to find a set $\mathcal{N}$ of neighboring records, each having its spatial distance to $\theta$ smaller than $\epsilon_{s}$ and temporal 
distance to $\theta$ smaller than $\epsilon_{t}$. In addition, the function GetAdaptivePtm (lines 6 and 18) computes an adaptive $p t_{m}$ associated with the current record.

To be specific, GetAdaptivePtm works as follows (lines 35-38). It first retrieves a set $\mathcal{N}_{t}$ of records within the time window $\left[t-\epsilon_{t}, t+\epsilon_{t}\right]$ by a temporal range query on $\Theta_{o}$ (line 36). Note that $\left|\mathcal{N}_{t}\right|$ is proportional to the local sampling rate $s t_{\text {local }}$ such that $\left|\mathcal{N}_{t}\right|=2 * \epsilon_{t} * s t_{\text {local }}$. Afterward, a rounddown sigmoid function $\left\lfloor\frac{e^{\left|N_{t}\right|-N}}{1+e^{\left|N_{t}\right|-N}} * 2 P+B\right\rfloor$ is used to obtain the adaptive $p t_{m}$ (line 37). In particular, $N, P, B$ are three integer parameters, and $p t_{m}$ increases monotonically with $\left|\mathcal{N}_{t}\right|$ from $B$ to $2 P+B$ and equals to $P+B$ when $\left|\mathcal{N}_{t}\right|=N$. The optimal values of parameters $\epsilon_{s}, \epsilon_{t}, N$, $P$, and $B$ can be determined by a grid search. The adaptive function here can be replaced with another design, such as one that considers both the sampling rate and potentially located region.

When the clustering is done, each cluster is converted into a dense snippet snpt and added to $\mathcal{A}_{\text {snpt }}$ (lines 20-23). After that, the algorithm iterates through each consecutive dense snippets $\left\langle s n p t_{i}, s_{n} t_{i+1}\right\rangle$ in $\mathcal{A}_{\text {snpt }}$ (lines 24-33). It first checks if the two dense snippets $s n p t_{i}$, snpt $t_{i+1}$ can be merged in the sense of the merging conditions defined on $\Delta_{t}$ and $\Delta_{s}$ (line 25). If it is, they are removed from $\mathcal{A}_{\text {snpt }}$ (line 26), merged into one (line 27), and added back to $\mathcal{A}_{\text {snpt }}$ (line 28). Otherwise, it further checks if there exists any positioning record labeled with noise between the two snippets (lines 29 and 30). If any of such record can be found, a non-dense snippet is formed by retrieving all such records in between, then added to $\mathcal{A}_{\text {snpt }}$ (lines 31-33). At the end, the timeordered sequence $\mathcal{A}_{\text {snpt }}$ is returned (line 34 ).

\section{A.2 Semantic Matching Algorithm}

Algorithm 5 uses the $\mathcal{E}$-function and the semantic region graph to translate a snippet $\Theta_{o}^{*}$ into a sequence $\Lambda_{o}^{*}$ of m-semantics. At the beginning, a time-ordered sequence $\Lambda_{o}^{*}$ is initialized to hold the matched $\mathrm{m}$-semantics (line 1 ). If the snippet is classified as stay by the function $\mathcal{E}\left(\Theta_{o}^{*}\right)$ (line 2), it is matched to a stay m-semantics (lines 2-9). In particular, the event and temporal annotations are made at first (line 3). Subsequently, the algorithm iterates through each positioning records $\theta_{i}$ and computes its location estimate confidence conf $f^{(i)}$ (lines 4-6). As a result, the stay position is inferred as $\hat{l}$ (line 7) and the spatial annotation $r$ is matched by searching on $G_{R}$ (line 8). Afterward, the matched $\mathrm{m}$-semantics $\lambda$ is added to $\Lambda_{o}^{*}$ (line 9).

Otherwise, if the snippet is corresponding to a pass event, we conduct the matching as follows (lines 10-20). First, an event annotation pass is made for each m-semantics to be matched, and a variable $\lambda^{\prime}$ is used to control the merge of consecutive pass m-semantics (line 11). The algorithm then iterates through each positioning record $\theta_{i}$ (lines 12-20). Specifically, the temporal annotation is generated (line 13) and the spatial annotation is determined as the region that contains $\theta_{i} . l$ (line 14). If the current $\theta_{i}$ 's spatial annotation is the same as $\lambda^{\prime} . r$, they are merged together (lines 15 and 16). Otherwise, $\lambda^{\prime}$ can no longer be merged with any subsequent $\mathrm{m}$-semantics and it is added to $\Lambda_{o}^{*}$, and the current matched annotations $(r, \tau, \delta)$ are assigned to $\lambda^{\prime}$ (lines 17-19). Note that $\lambda^{\prime}$ should be added to $\Lambda_{o}^{*}$ in the last loop of iteration (line 20). Finally, $\Lambda_{o}^{*}$ is returned as a sequence of matched m-semantics (line 21).

\section{A.3 Mobility Knowledge Construction Algorithm}

Algorithm 6 takes $G_{R}$ and a set of ms-sequences as input and returns the mobility knowledge in a hash table $\mathcal{M K}$. At the beginning, the hash table $\mathcal{M} \mathcal{K}$ is initialized to store the path set $\Phi$ and the corresponding transition probabilities $T P$ for each directed pair of stay regions (line 1). For each such pair $\left\langle r_{s}^{\prime \prime}, r_{e}^{\prime \prime}\right\rangle$, a function ConstructForOnePair is called to obtain the corresponding $\Phi$ and TP (lines 2 and 3). 


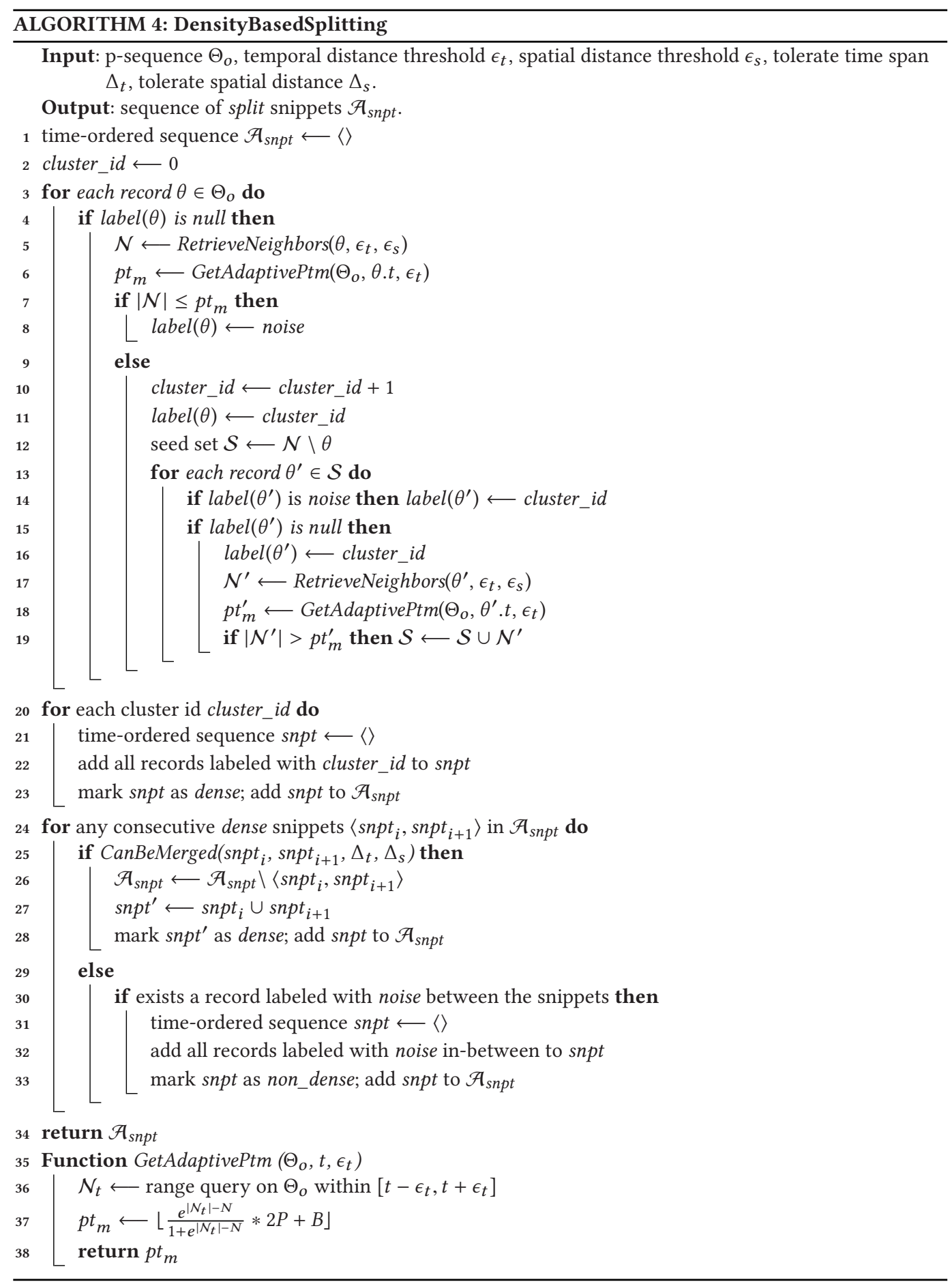




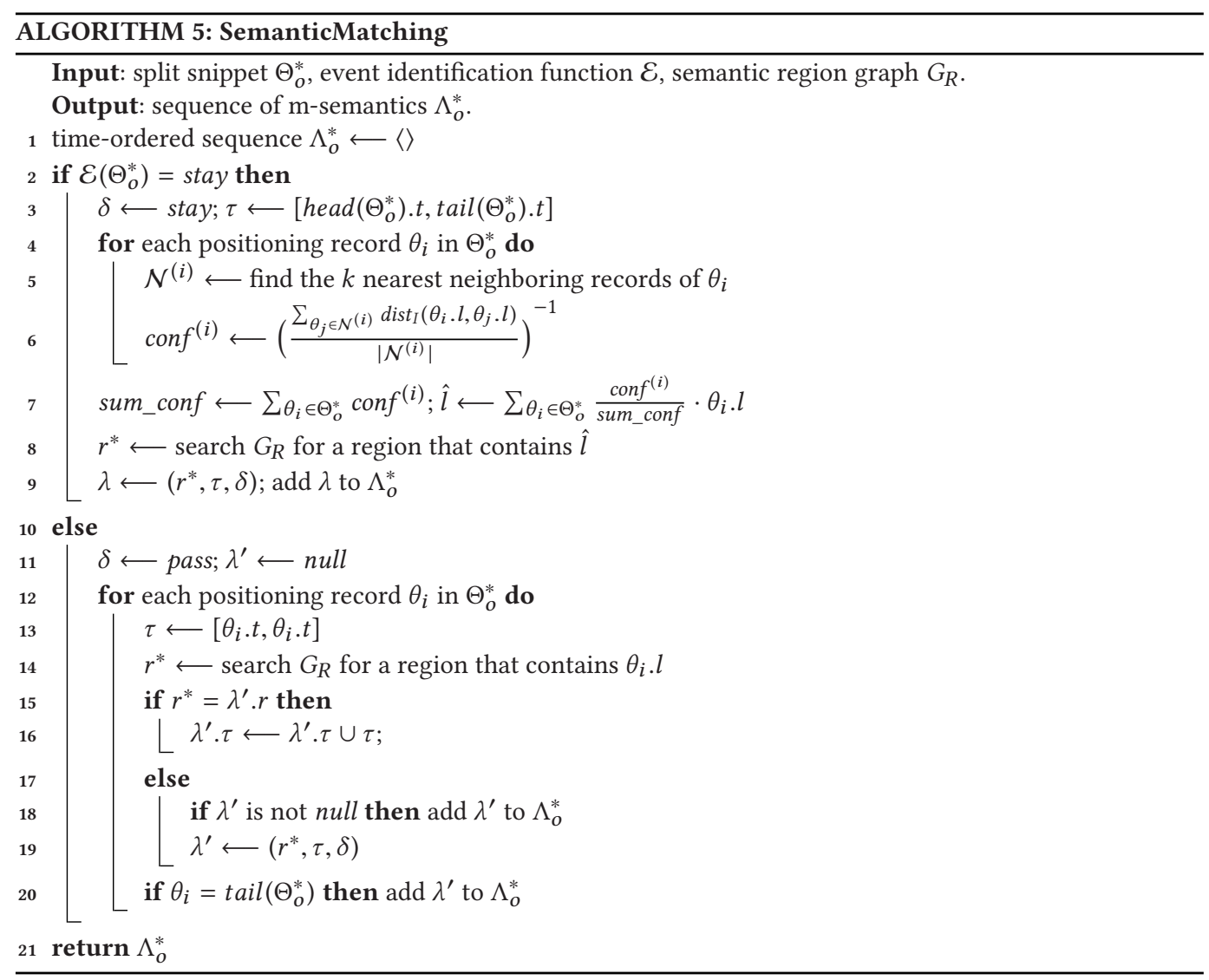

Function ConstructForOnePair works as follows (lines 5-24). First, it performs an $A *-$ Search to find a set $\Phi$ of candidate paths (line 6) and constructs the transition probabilities TP for each two directly connected regions in the candidate paths (lines 7-23). In particular, a hash table $\mathcal{H}_{P T}$ that records the region patterns is constructed by processing each ms-sequence $\Lambda_{o} \in \mathcal{S}_{\Lambda}$ (lines 8-12). Afterward, it records the score for each pair of directly connected regions in a candidate path such that a hash table $\mathcal{H}_{s}$ is constructed (lines 13-19). Consequently, the transition probabilities TP is computed with the scores recorded in $\mathcal{H}_{s}$ (lines 20-23). At the end, the candidate path set $\Phi$ is returned along with TP (line 24).

\section{A.4 M-Semantics Inference Algorithm}

Algorithm 7 gives the procedure of inferring the missing $\mathrm{m}$-semantics for an original ms-sequence by utilizing the mobility knowledge $\mathcal{M K}$ and the indoor mobility constraints captured in $G_{R}$. Given an observation $\left\langle\lambda_{s}^{\prime \prime}, \ldots, \lambda_{e}^{\prime \prime}\right\rangle$ between stay m-semantics $\lambda_{s}^{\prime \prime}$ and $\lambda_{e}^{\prime \prime}$ (line 1), the corresponding candidate path set $\Phi$ is loaded from $\mathcal{M K}\left[\left\langle r_{s}^{\prime \prime}, r_{e}^{\prime \prime}\right\rangle\right]$ (line 2), and a function InferMostLikelyPath is called to infer a most-likely path $\hat{\phi} \in \Phi$ for the observation between $\lambda_{s}^{\prime \prime}$ and $\lambda_{e}^{\prime \prime}$ (line 3 ). Furthermore, the algorithm makes use of the GRDs between directly connected regions to infer the time period for each region $r_{x}$ contained in $\hat{\phi}$ (lines 4-10). In particular, a function InferDurationTime is called to infer the time period $\tau_{x}$ for each $r_{x}$ (line 5). If the current $r_{x}$ has been observed in an m-semantics $\lambda_{x}$ in $\Lambda_{o}$ (line 6), its inferred time period is merged with $\lambda_{x}$ to form a new m-semantics, and the 


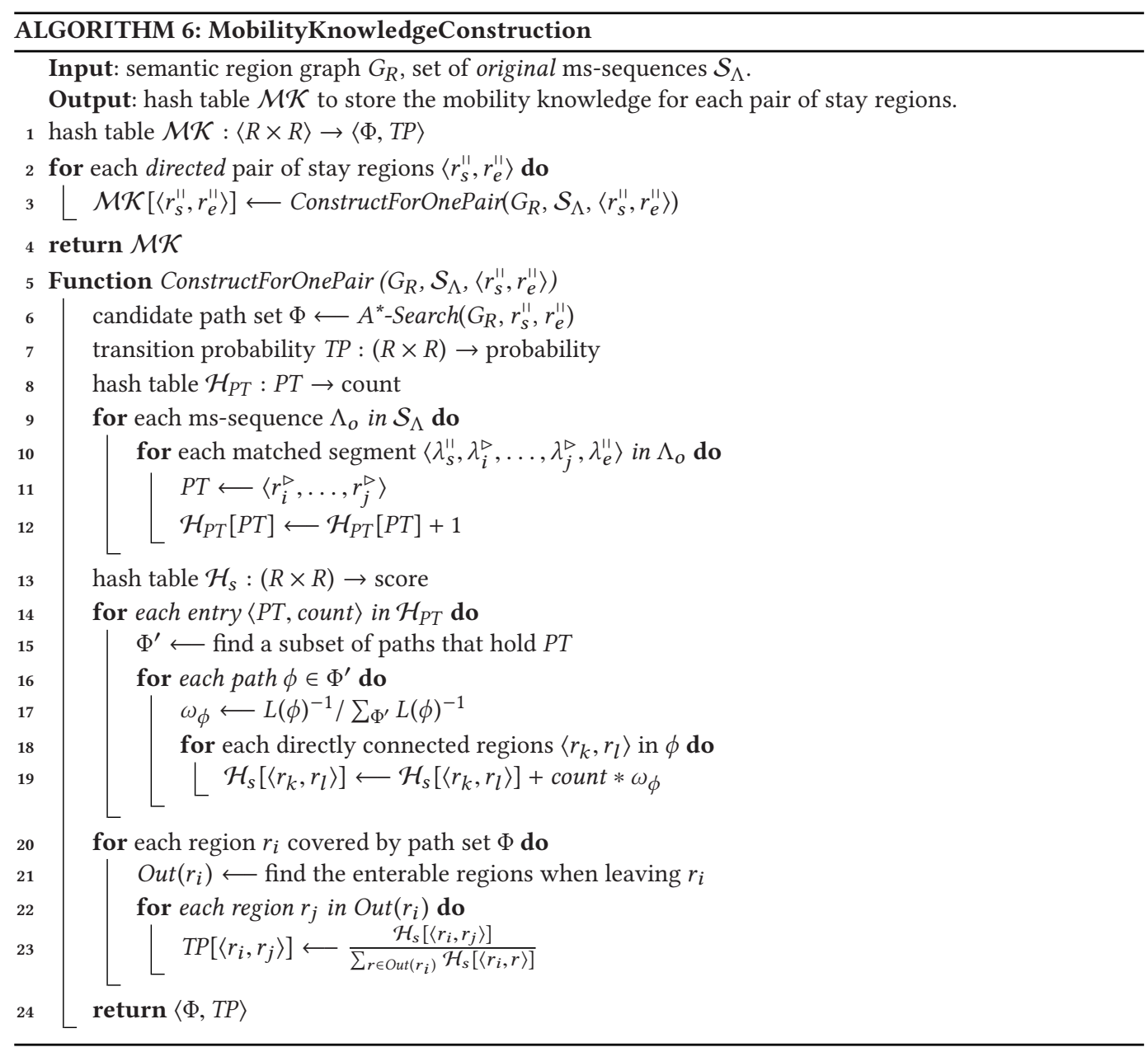

new m-semantics is added back to $\Lambda_{o}$ (lines 7 and 8); otherwise, the algorithm maps $r_{x}$ to a pass m-semantics and adds it to $\Lambda_{o}$ (line 10). At the end, the complemented ms-sequence $\Lambda_{o}$ is returned (line 11). The technical details of function InferMostLikelyPath and function InferDurationTime have been given in Section 5.2.1 and Section 5.2.2 of the article, respectively.

\section{B FORMALIZATION DETAILS OF M-SEMANTICS INFERENCE}

\section{B.1 The Length of an Indoor Candidate Path}

Lemma B.1. Given an indoor path $\phi=r_{i} \rightarrow \ldots, \rightarrow r_{j}$, its path length $L(\phi)=\sum_{k=i}^{j} \operatorname{dist}_{g r}\left(r_{k}, r_{k+1}\right)$ is an upper bound of the minimum distance required to ensure any object can reach $r_{j}$ from $r_{i}$ through the path $\phi$.

Proof. Suppose that a region $r_{1}$ connects with region $r_{2}$ and the path corresponding to the GRD from $r_{1}$ to $r_{2}$ begins at a position $l_{1}^{(s)} \in r_{1}$ and ends at a position $l_{2}^{(e)} \in r_{2}$. In addition, $r_{2}$ connects with region $r_{3}$ and the path corresponding to the GRD from $r_{2}$ to $r_{3}$ begins at a position $l_{2}^{(s)} \in r_{2}$ and ends at a position $l_{3}^{(e)} \in r_{3}$. Suppose that an object $o$ is at the farthest position $l_{1}^{\prime} \in r_{1}$ from region $r_{3}$ and it goes through $r_{2}$ to reach $r_{3}$ at a position $l_{3}^{\prime} \in r_{3}$. The corresponding traveling distance 


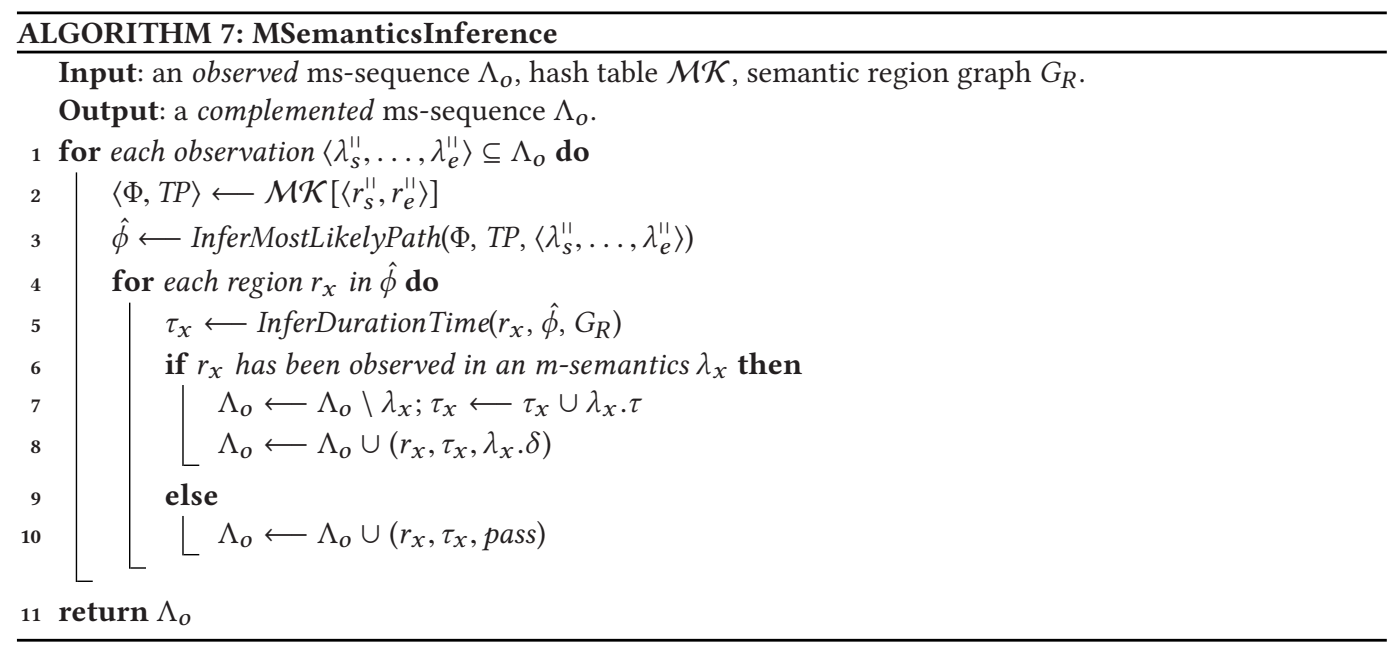

is $\operatorname{dist}_{I}\left(l_{1}^{\prime}, l_{2}^{\prime}\right)+\operatorname{dist}_{I}\left(l_{2}^{\prime}, l_{3}^{\prime}\right)$, where $l_{2}^{\prime} \in r_{2}$ is a position where $o$ went out of $r_{1}$ and entered into $r_{2}$. According to the definition of GRD, we have $\forall l_{1} \in r_{1}$, $\operatorname{dist}_{I}\left(l_{1}, l_{2}^{(e)}\right) \leq \operatorname{dist}_{I}\left(l_{1}^{(s)}, l_{2}^{(e)}\right)$ and $\forall l_{2} \in$ $r_{2}$, $\operatorname{dist}_{I}\left(l_{2}, l_{3}^{(e)}\right) \leq \operatorname{dist}_{I}\left(l_{2}^{(s)}, l_{3}^{(e)}\right)$. Consequently, we have $\operatorname{dist}_{I}\left(l_{1}^{\prime}, l_{2}^{\prime}\right)+\operatorname{dist}_{I}\left(l_{2}^{\prime}, l_{3}^{\prime}\right) \leq \operatorname{dist}_{I}\left(l_{1}^{\prime}, l_{2}^{(e)}\right)+$ $\operatorname{dist}_{I}\left(l_{2}^{\prime}, l_{3}^{(e)}\right) \leq \operatorname{dist}_{I}\left(l_{1}^{(s)}, l_{2}^{(e)}\right)+\operatorname{dist}_{I}\left(l_{2}^{(s)}, l_{3}^{(e)}\right)=\operatorname{dist}_{g r}\left(r_{1}, r_{2}\right)+\operatorname{dist}_{g r}\left(r_{2}, r_{3}\right)$.

In a more general case, given an indoor path $r_{i} \rightarrow \ldots, \rightarrow r_{j}$, we have the minimum required distance that ensures an object can reach $r_{j}$ from $r_{i}$ through the path is no greater than $\sum_{k=i}^{j} \operatorname{dist}_{g r}\left(r_{k}, r_{k+1}\right)$. The lemma is proved.

\section{B.2 Posterior Probability of an Indoor Candidate Path}

Given an observed region pattern $P T^{(o)}$ and a candidate path $\phi=r_{s}^{\prime \prime} \rightarrow r_{a}^{\triangleright} \rightarrow \cdots \rightarrow r_{b}^{\triangleright} \rightarrow r_{q}^{\triangleright} \rightarrow$ $r_{c}^{\triangleright} \rightarrow \cdots \rightarrow r_{d}^{\triangleright} \rightarrow r_{e}^{\prime \prime}$, we have the posterior probability $\mathrm{P}\left(\phi \mid P T^{(o)}\right)$ in the context of a first-order Markov stochastic process [30] as

$$
\begin{aligned}
\mathrm{P}\left(\phi \mid P T^{(o)}\right) & =\mathrm{P}\left(r_{s}^{\prime \prime}, r_{a}^{\triangleright}, \ldots, r_{b}^{\triangleright}, r_{q}^{\triangleright}, r_{c}^{\triangleright}, \ldots, r_{d}^{\triangleright}, r_{e}^{\prime \prime} \mid r_{s}^{\prime \prime}, r_{q}^{\triangleright}, r_{e}^{\prime \prime}\right) \\
& =\mathrm{P}\left(r_{s}^{\prime \prime}, r_{a}^{\triangleright}, \ldots, r_{b}^{\triangleright}, r_{q}^{\triangleright} \mid r_{s}^{\prime \prime}, r_{q}^{\triangleright}\right) \cdot \mathrm{P}\left(r_{q}^{\triangleright}, r_{c}^{\triangleright}, \ldots, r_{d}^{\triangleright}, r_{e}^{\prime \prime} \mid r_{q}^{\triangleright}, r_{e}^{\prime \prime}\right) \\
& =\frac{\mathrm{P}\left(r_{q}^{\triangleright} \mid r_{b}^{\triangleright}\right) \prod_{x=a}^{b-1} \mathrm{P}\left(r_{x+1}^{\triangleright} \mid r_{x}^{\triangleright}\right) \mathrm{P}\left(r_{a}^{\triangleright} \mid r_{s}^{\prime \prime}\right) \mathrm{P}\left(r_{s}^{\prime \prime}\right)}{\mathrm{P}\left(r_{s}^{\prime \prime}\right) \mathrm{P}\left(r_{q}^{\triangleright}\right)} \cdot \frac{\mathrm{P}\left(r_{e}^{\prime \prime} \mid r_{d}^{\triangleright}\right) \prod_{y=c}^{d-1} \mathrm{P}\left(r_{y+1}^{\triangleright} \mid r_{y}^{\triangleright}\right) \mathrm{P}\left(r_{c}^{\triangleright} \mid r_{q}^{\triangleright}\right) \mathrm{P}\left(r_{q}^{\triangleright}\right)}{\mathrm{P}\left(r_{q}^{\triangleright}\right) \mathrm{P}\left(r_{e}^{\prime \prime}\right)} \\
& =\frac{\mathrm{P}\left(r_{q}^{\triangleright} \mid r_{b}^{\triangleright}\right) \prod_{x=a}^{b-1} \mathrm{P}\left(r_{x+1}^{\triangleright} \mid r_{x}^{\triangleright}\right) \mathrm{P}\left(r_{a}^{\triangleright} \mid r_{s}^{\prime \prime}\right)}{\mathrm{P}\left(r_{q}^{\triangleright}\right)} \cdot \frac{\mathrm{P}\left(r_{e}^{\prime \prime} \mid r_{d}^{\triangleright}\right) \prod_{y=c}^{d-1} \mathrm{P}\left(r_{y+1}^{\triangleright} \mid r_{y}^{\triangleright}\right) \mathrm{P}\left(r_{c}^{\triangleright} \mid r_{q}^{\triangleright}\right)}{\mathrm{P}\left(r_{e}^{\prime \prime}\right)} \\
& \propto \mathrm{P}\left(r_{q}^{\triangleright} \mid r_{b}^{\triangleright}\right) \prod_{x=a}^{b-1} \mathrm{P}\left(r_{x+1}^{\triangleright} \mid r_{x}^{\triangleright}\right) \mathrm{P}\left(r_{a}^{\triangleright} \mid r_{s}^{\prime \prime}\right) \cdot \mathrm{P}\left(r_{e}^{\prime \prime} \mid r_{d}^{\triangleright}\right) \prod_{y=c}^{d-1} \mathrm{P}\left(r_{y+1}^{\triangleright} \mid r_{y}^{\triangleright}\right) \mathrm{P}\left(r_{c}^{\triangleright} \mid r_{q}^{\triangleright}\right) .
\end{aligned}
$$

\section{ADDITIONAL EXPERIMENTAL RESULTS}

\section{C.1 Answering Queries Using M-Semantics on Real Data}

In addition to the query time interval QT we evaluated in Section 6.1.5, we also investigate the effects of other parameters related to the two top- $k$ queries, namely $k$ and the query set size $|Q|$. 
Table 5. Parameter Settings on Real Data

\begin{tabular}{cc}
\hline Parameters & Settings \\
\hline Query type & TkPRQ, TkFRPQ \\
QT (minutes) & $60, \mathbf{1 2 0}, 180,240$ \\
$k$ & $20,40, \mathbf{6 0}, 80$ \\
$|Q|$ (\% of semantic regions) & $30 \%, \mathbf{5 0} \%, 70 \%$ \\
\hline
\end{tabular}

The parameter settings are shown in Table 5, where default values are in bold. We test each parameter with others fixed to defaults. The experimental evaluations focus on the search effectiveness in terms of the metric precision (see Section 6.1.5).

Effect of $k$. We fix $|Q|=202 \times 50 \%=101$ and QT $=120$ minutes, and vary $k$ from 20 to 80 . The results for TkPRQ and TkFRPQ are reported in Figure 16(a) and (b), respectively. As shown in Figure 16(a), the precisions of all search methods increase moderately with an increasing $k$. Since $|Q|$ is fixed in our test, a larger $k$ tends to include more query regions in the top- $k$ search results and therefore the precision improves in all of the methods. Clearly, IMS-CAC performs the best in each $k$ values; its precision stays higher than 0.84 with $k$ up to 60 . The results also verify the effectiveness of our cleaning method, as the search methods with the cleaning (i.e., RAW-C, IMSCA, and IMS-CAC) clearly outperform other alternatives.
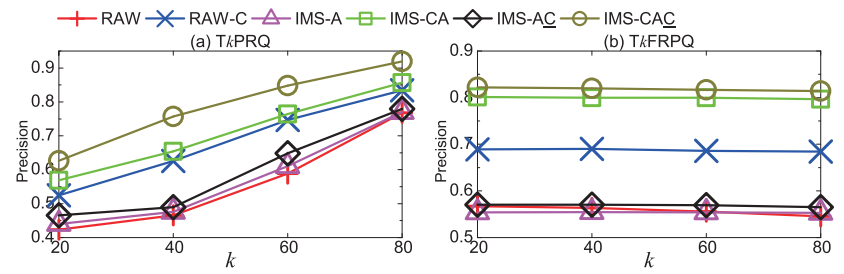

Fig. 16. Query answering effectiveness vs. $k$ on real data.

However, when we increase $k$, the precisions of all search methods stay very stable in processing the TkFRPQ query. Different from the TkPRQ, TkFRPQ needs to find $k$ frequent region pairs from ${ } 2^{Q} \mid$ candidate region pairs, whose number is significantly larger than that of the candidate regions in T $k$ PRQ. In such a case, increasing $k$ from 20 to 60 does not affect the precisions in all methods, as $k$ is relatively small compared to $\left|2^{Q}\right|$. Nevertheless, the m-semantics constructed by IMS-CAC are still the best in answering the TkFRPQ in different $Q$ settings.

Effect of $|Q|$. We also very $|Q|$ from $30 \%$ to $70 \%$ with other parameters fixed by default. The results are reported in Figure 17. Referring to Figure 17(a), increasing $|Q|$ deteriorates the TkPRQ's precision in all of the methods as more query regions need to be computed and ranked. However, the precision of our overall framework IMS-CAC decreases slightly compared to other alternatives.
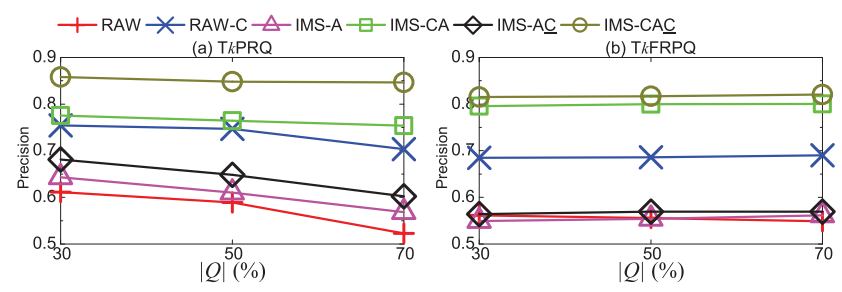

Fig. 17. Query answering effectiveness vs. $|Q|$ on real data. 
This shows that its constructed $m$-semantics are very effective to answer the top- $k$ frequent region query. When increasing $|Q|$, the precisions of those methods without data cleaning decreases more rapidly than the alternatives that process on the cleaned data.

However, TkFRPQ's precision in each method is also insensitive to an increasing $|Q|$. In the shopping mall where our data was collected, the most frequent region pairs are always among the most popular stores visited by the shoppers. Hence, involving more semantic regions will not affect the returned results when $k$ is fixed in the TkFRPQ query.

In general, the precision results on varying $k$ and $|Q|$ demonstrate that the m-semantics constructed by our framework IMS-CAC are very effective in answering the two indoor top- $k$ queries.

\section{C.2 Effectiveness of Raw Data Cleaning on Synthetic Data}

According to the ground truth trajectory recorded for each object, here we study the effectiveness of our proposed raw data cleaning method on the synthetic data.

Alternative methods. To verify the effectiveness of using the indoor mobility constraints, we consider two alternatives, namely the ED method that uses the Euclidean distance to represent the speed constraint as well as interpolate the new location estimates, and the MIWD method that uses the MIWD instead. We apply different speed threshold $v_{m}$ to ED and MIWD, and compare them with the original raw p-sequence without the cleaning (denoted as Original).

Performance metrics. We measure the effectiveness of ED and MIWD in two aspects. On the one hand, given a p-sequence $\Theta$ and its ground truth $\Theta_{g}$, we define $\Theta$ 's floor accuracy as the fraction of its records having a correct floor value with respect to the ground truth. On the other hand, excluding those records that have false floor values, we define $\Theta$ 's average location error (ALE) as

$$
A L E=\sum_{\theta \in \Theta, \theta_{g} \in \Theta_{g}, \theta . t=\theta_{g} . t, \theta . l . f=\theta_{g} . l . f} \frac{\operatorname{dist}_{I}\left(\theta . l, \theta_{g} \cdot l\right)}{|\Theta|} .
$$

For each synthetic IPT instance (see Table 4 in the main article), we measure the average value of floor accuracies and ALEs of all p-sequences. We vary and test different settings of the maximum positioning period $T$ and the positioning error factor $\mu$.

Effect of T. First, we fix $\mu=3 m$ and vary $T$ from $5 s$ to $15 \mathrm{~s}$. Referring to Figure 18(a), in each setting of $T$, the floor accuracy staying around 0.7 in the original p-sequence is improved significantly by the MIWD-based methods. When increasing $T$, both methods' floor accuracy decreases, but MIWD's decreases slower than that of ED. The decline is due to that the time difference between consecutive records becomes larger and the speed checking tends to be less reliable. Nevertheless, when $T=15 s$, MIWD still achieves a floor accuracy of around 0.9. However, MIWD beats ED in all tests when they use the same $v_{m}$. Interestingly, when we set $v_{m}$ to $1.9 \mathrm{~m} / \mathrm{s}$ and vary $T$ from $10 \mathrm{~s}$ to $15 \mathrm{~s}$, both methods' floor accuracies increase, showing that a tighter speed constraint is better to capture object movements when data is sparser.
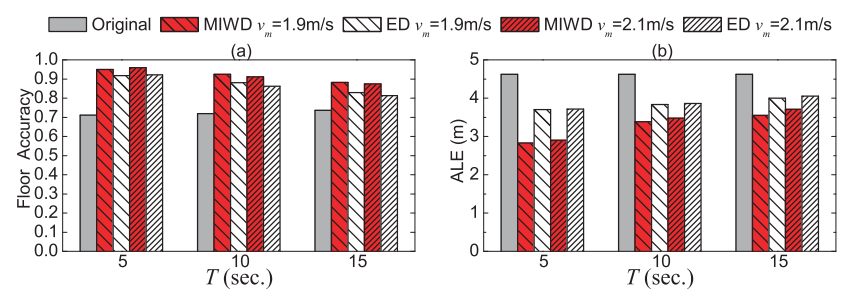

Fig. 18. Cleaning effectiveness vs. $T$ on synthetic data. 


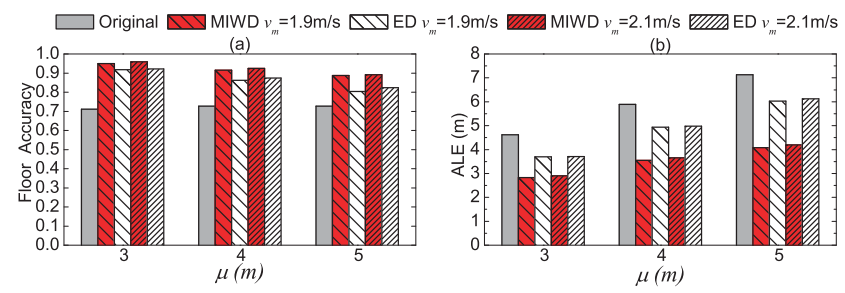

Fig. 19. Cleaning effectiveness vs. $\mu$ on synthetic data.

Referring to Figure 18(b), the ALE of the original p-sequence is also reduced clearly by our cleaning methods. The reduction decreases but at a slow pace when a larger $T$ is involved. Still, MIWD beats ED in all tests when we vary $T$.

The results reported on both measures verify that our mobility constraint based on MIWD is very effective in cleaning the raw indoor positioning data even when the data is temporally sparse.

Effect of $\mu$. We vary and test the positioning error factor $\mu$ from $3 m$ to $5 m$ with $T$ fixed to $5 s$. Referring to Figure 19(a), the floor accuracy is always improved significantly in different settings of $\mu$. In addition, MIWD's improvement only decreases slightly when $\mu$ increases. As reported in Figure 19(b), the ALE is also reduced clearly by the cleaning methods with different $\mu \mathrm{s}$, and the reduction is more significant when $\mu$ increases. In both two measures, MIWD performs better than ED, showing it has a better capability to identify the errors and interpolate new location estimates.

To sum up, the results in different settings of $T$ and $\mu$ verify that our indoor mobility constraintbased cleaning method using MIWD is still effective when the temporal sparsity and positioning errors are involved in the raw positioning data.

\section{REFERENCES}

[1] IIoT World. 2018. Infographic: Top Industries Using Indoor Positioning. Retrieved September 11, 2020 from https://iiot-world.com/connected-industry/top-industries-using-indoor-positioning/.

[2] Wikipedia. n.d. List of Countries by Smartphone Penetration: 2018 Rankings. Retrieved September 11, 2020 from https://en.wikipedia.org/wiki/List_of_countries_by_smartphone_penetration\#2018_rankings.

[3] Smile. n.d. Smile-Statistical Machine Intelligence and Learning Engine. Retrieved September 11, 2020 from https://haifengl.github.io.

[4] L. O. Alvares, V. Bogorny, B. Kuijpers, J. de Macedo, B. Moelans, and A. Vaisman. 2007. A model for enriching trajectories with semantic geographical information. In Proceedings of the 15th Annual ACM International Symposium on Advances in Geographic Information Systems (GIS'07). Article 22, 8 pages.

[5] A. I. Baba, M. Jaeger, H. Lu, T. B. Pedersen, W.-S. Ku, and X. Xie. 2016. Learning-based cleaning for indoor RFID data. In Proceedings of the 2016 International Conference on Management of Data (SIGMOD'16). 925-936.

[6] A. I. Baba, H. Lu, T. B. Pedersen, and X. Xie. 2014. Handling false negatives in indoor RFID data. In Proceedings of IEEE Conference on Mobile Data Management (MDM'14). 117-126.

[7] A. I. Baba, H. Lu, X. Xie, and T. B. Pedersen. 2013. Spatiotemporal data cleansing for indoor RFID tracking data. In Proceedings of IEEE Conference on Mobile Data Management (MDM'13). 187-196.

[8] D. Birant and A. Kut. 2007. ST-DBSCAN: An algorithm for clustering spatial-temporal data. Data \& Knowledge Engineering 60, 1 (2007), 208-221.

[9] A. Blum and T. Mitchell. 1998. Combining labeled and unlabeled data with co-training. In Proceedings of the 11th Annual Conference on Computational Learning Theory (COLT'98). 92-100.

[10] X. Cao, G. Cong, and C. S. Jensen. 2010. Mining significant semantic locations from GPS data. Proceedings of the VLDB Endowment 3, 1 (2010), 1009-1020.

[11] H. Chen, W.-S. Ku, H. Wang, and M.-T. Sun. 2010. Leveraging spatio-temporal redundancy for RFID data cleaning. In Proceedings of the 2010 International Conference on Management of Data (SIGMOD'10). 51-62.

[12] J. Chen and M. Bierlaire. 2015. Probabilistic multimodal map matching with rich smartphone data. fournal of Intelligent Transportation Systems 19, 2 (2015), 134-148.

[13] B. Fang, S. Liao, K. Xu, H. Cheng, C. Zhu, and H. Chen. 2012. A novel mobile recommender system for indoor shopping. Expert Systems with Applications 39, 15 (2012), 11992-12000. 
[14] V. Fox, J. Hightower, L. Liao, D. Schulz, and G. Borriello. 2003. Bayesian filtering for location estimation. Pervasive Computing 2, 3 (2003), 24-33.

[15] A. Globerson and T. S. Jaakkola. 2008. Fixing max-product: Convergent message passing algorithms for MAP LPrelaxations. In Advances in Neural Information Processing Systems. 553-560.

[16] R. H. Güting, F. Valdés, and M. L. Damiani. 2015. Symbolic trajectories. ACM Transactions on Spatial Algorithms and Systems 1, 2 (2015), 7.

[17] V. Honkavirta, T. Perala, S. Ali-Loytty, and R. Piché. 2009. A comparative survey of WLAN location fingerprinting methods. In Proceedings of the 2009 6th Workshop on Positioning, Navigation, and Communication (WPNC'09). 243-251.

[18] J. Hightower and G. Borriello. 2001. Location systems for ubiquitous computing. IEEE Computer 34, 8 (2001), 57-66.

[19] P. L. Jenkins, T. J. Phillips, E. J. Mulberg, and S. P. Hui. 1992. Activity patterns of Californians: Use of and proximity to indoor pollutant sources. Atmospheric Environment 26, 12 (1992), 2141-2148.

[20] D. B. Johnson and D. A. Maltz. 1996. Dynamic source routing in ad hoc wireless networks. In Mobile Computing. Springer, 153-181.

[21] N. Klepeis, W. Nelson, W. Ott, J. Robinson, A. Tsang, P. Switzer, J. Behar, S. Hern, and W. Engelmann. 2001. The national human activity pattern survey (NHAPS): A resource for assessing exposure to environmental pollutants. fournal of Exposure Science 11, 3 (2001), 231.

[22] M. B. Kjærgaard, M. Wirz, D. Roggen, and G. Tröster. 2012. Mobile sensing of pedestrian flocks in indoor environments using WIFI signals. In Proceedings of the 2012 IEEE International Conference on Pervasive Computing and Communications (PerCom'12). 95-10.

[23] H. Li, H. Lu, X, Chen, G. Chen, K. Chen, and L. Shou. 2016. Vita: A versatile toolkit for generating indoor mobility data for real-world buildings. Proceedings of the VLDB Endowment 9, 13 (2016), 1453-1456.

[24] H. Li, H. Lu, F. Shi, G. Chen, K. Chen, and L. Shou. 2018. TRIPS: A system for translating raw indoor positioning data into visual mobility semantics. Proceedings of the VLDB Endowment 11, 12 (2018), 1918-1921.

[25] H. Li, H. Lu, L. Shou, G. Chen, and K. Chen. 2018. Finding most popular indoor semantic locations using uncertain mobility data. IEEE Transactions on Knowledge and Data Engineering 31, 11 (2018), 2108-2123.

[26] H. Li, H. Lu, L. Shou, G. Chen, and K. Chen. 2018. In search of indoor dense regions: An approach using indoor positioning data. IEEE Transactions on Knowledge and Data Engineering 30, 8 (2018), 1481-1495.

[27] L. Liao, D. Fox, and H. Kautz. 2006. Location-based activity recognition. In Advances in Neural Information Processing Systems. 787-794.

[28] H. Lu, X. Cao, and C. S. Jensen. 2012. A foundation for efficient indoor distance-aware query processing. In Proceedings of the 28th International Conference on Data Engineering (ICDE'12). 438-449.

[29] H. Lu, C. Guo, B. Yang, and C. S. Jensen. 2016. Finding frequently visited indoor pois using symbolic indoor tracking data. In Proceedings of the 19th International Conference on Extending Database Technology (EDBT'16). 449-460.

[30] X. Lu, E. Wetter, N. Bharti, A. J. Tatem, and L. Bengtsson. 2013. Approaching the limit of predictability in human mobility. Scientific Reports 3, 10 (2013), 2923.

[31] G. Marketos, E. Frentzos, I. Ntoutsi, N. Pelekis, A. Raffaetà, and Y. Theodoridis. 2008. Building real-world trajectory warehouses. In Proceedings of the 7th ACM International Workshop on Data Engineering for Wireless and Mobile Access (MobiDE'08). 8-15.

[32] T. P. Nogueira, R. B. Braga, C. T. de Oliveira, and H. Martin. 2018. FrameSTEP: A framework for annotating semantic trajectories based on episodes. Expert Systems with Applications 92 (2018), 533-545.

[33] C. Parent, S. Spaccapietra, C. Renso, G. Andrienko, N. Andrienko, V. Bogorny, M. L. Damiani, et al. 2013. Semantic trajectories modeling and analysis. ACM Computing Surveys 45, 4 (2013), Article 42.

[34] T. S. Prentow, H. Blunck, K. Grønbæk, and M. B. Kjærgaard. 2014. Estimating common pedestrian routes through indoor path networks using position traces. In Proceedings of IEEE Conference on Mobile Data Management (MDM'14). 43-48.

[35] T. S. Prentow, A. Thom, H. Blunck, and J. Vahrenhold. 2015. Making sense of trajectory data in indoor spaces. In Proceedings of IEEE Conference on Mobile Data Management (MDM'15). 116-121.

[36] S.-Y. Teng, W.-S. Ku, and K.-T. Chuang. 2017. Toward mining stop-by behaviors in indoor space. ACM Transactions on Spatial Algorithms and Systems 3, 2 (2017), Article 7.

[37] S. Scellato, M. Musolesi, C. Mascolo, V. Latora, and A. T. Campbell. 2011. NextPlace: A spatio-temporal prediction framework for pervasive systems. In Proceedings of the International Conference on Pervasive Computing (ICPC'11). $152-169$.

[38] N. Schuessler and K. W. Axhausen. 2009. Processing raw data from global positioning systems without additional information. Transportation Research Record 2105, 1 (2009), 28-36.

[39] H. Su, K. Zheng, K. Zeng, J. Huang, S. Sadiq, N. J. Yuan, and X. Zhou. 2015. Making sense of trajectory data: A partitionand-summarization approach. In Proceedings of the 31st International Conference on Data Engineering (ICDE'15). 963974. 
[40] L. Wei, Y. Zheng, and W. Peng. 2012. Constructing popular routes from uncertain trajectories. In Proceedings of the 18th ACM SIGKDD International Conference on Knowledge Discovery and Data Mining (KDD'12). 195-203.

[41] F. Wu and Z. Li. 2016. Where did you go: Personalized annotation of mobility records. In Proceedings of the 25th ACM International Conference on Information and Knowledge Management (CIKM'16). 589-598.

[42] F. Wu, Z. Li, W.-C. Lee, H. Wang, and Z. Huang. 2015. Semantic annotation of mobility data using social media. In Proceedings of the 24th International Conference on World Wide Web (WWW'15). 1253-1263.

[43] X. Xie, H. Lu, and T. B. Pedersen. 2013. Efficient distance-aware query evaluation on indoor moving objects. In Proceedings of the 29th International Conference on Data Engineering (ICDE'13). 434-445.

[44] Z. Yan, D. Chakraborty, C. Parent, S. Spaccapietra, and K. Aberer. 2013. Semantic trajectories: Mobility data computation and annotation. ACM Transactions on Intelligent Systems and Technology 4, 3 (2013), 49.

[45] K. Zheng, Y. Zheng, X. Xie, and X. Zhou. 2012. Reducing uncertainty of low-sampling-rate trajectories. In Proceedings of the 28th International Conference on Data Engineering (ICDE'12). 1144-1155.

[46] C. Zhang, J. Han, L. Shou, J. Lu, and T. La Porta. 2014. Splitter: Mining fine-grained sequential patterns in semantic trajectories. Proceedings of the VLDB Endowment 7, 9 (2014), 769-780.

[47] K. Zheng and X. Xie. 2011. Learning travel recommendations from user-generated GPS traces. ACM Transactions on Intelligent Systems and Technology 2, 1 (2011), 2.

[48] W. Zeng and R. L. Church. 2009. Finding shortest paths on real road networks: The case for A*. International fournal of GIS 23, 4 (2009), 531-543.

Received August 2019; revised November 2019; accepted January 2020 